\title{
Improved calculation of the isospin-symmetry-breaking corrections to superallowed Fermi $\beta$ decay
}

\author{
I. S. Towner* and J. C. Hardy \\ Cyclotron Institute, Texas A\&M University, College Station, Texas 77843, USA
}

(Received 16 October 2007; published 7 February 2008)

\begin{abstract}
We report new shell-model calculations of the isospin-symmetry-breaking correction $\delta_{C}$ to superallowed $0^{+} \rightarrow$ $0^{+}$nuclear $\beta$ decay. The most important improvement is the inclusion of core orbitals, which are demonstrated to have a significant impact on the mismatch in the radial wave functions of the parent and daughter states. We determine which core orbitals are important to include from an examination of measured spectroscopic factors in single-nucleon pickup reactions. In addition, where new sets of effective interactions have become available since our last calculation, we now include them; this leads to small changes in $\delta_{\mathrm{NS}}$ as well. We also examine the new radiative-correction calculation by Marciano and Sirlin and, by a simple reorganization, show that it is possible to preserve the conventional separation into a nucleus-independent "inner" radiative term, $\Delta_{R}^{V}$, and a nucleus-dependent "outer" term, $\delta_{R}^{\prime}$. We tabulate the new values for $\delta_{C}, \delta_{\mathrm{NS}}$, and $\delta_{R}^{\prime}$ for 20 superallowed transitions, including the 13 currently well-studied cases. With these new correction terms, the corrected $\mathcal{F} t$ values for the 13 cases are statistically consistent with one another, and the anomalousness of the ${ }^{46} \mathrm{~V}$ result disappears. These new calculations lead to a lower average $\overline{\mathcal{F} t}$ value and a higher value for $V_{\text {ud. }}$ The sum of squares of the top-row elements of the Cabibbo-Kobayashi-Maskawa matrix now agrees exactly with unitarity.
\end{abstract}

DOI: 10.1103/PhysRevC.77.025501

PACS number(s): 23.40.Bw, 23.40.Hc, 27.40.+z, 27.50.+e

\section{INTRODUCTION}

Superallowed $0^{+} \rightarrow 0^{+}$nuclear $\beta$ decay currently provides the most precise value for $V_{\mathrm{ud}}$, the up-down element of the Cabibbo-Kobayashi-Maskawa (CKM) matrix [1-3]. This element is the key ingredient of the most demanding available test of CKM-matrix unitarity, a fundamental requirement of the electroweak standard model. To extract $V_{\mathrm{ud}}$ from the experimental data, small theoretical corrections - of order $\sim 1 \%$ - must be applied to take account of unobserved radiative effects as well as the isospin symmetry breaking that occurs between the analog parent and daughter states of each superallowed transition [4,5]. Even though these corrections are very small, experimental measurements have by now reached such high precision that the uncertainty on $V_{\mathrm{ud}}( \pm 0.03 \%)$ is currently dominated not by experiment but by the uncertainty on these theoretical corrections.

In the determination of $V_{\mathrm{ud}}$, an important strength of the nuclear measurements is that many $0^{+} \rightarrow 0^{+}$transitions are available for study; currently, 13 transitions, ranging from ${ }^{10} \mathrm{C}$ to ${ }^{74} \mathrm{Rb}$, have been measured with high precision. With so many, it becomes possible to validate the analysis procedure by checking that all transitions individually yield statistically consistent results for $V_{\mathrm{ud}}$. Since the isospin-symmetrybreaking corrections depend on nuclear structure, they differ from transition to transition and are particularly sensitive to this consistency test. Thus the appearance of an anomalous result from any transition could signal a problem with the structure-dependent correction for that case, a problem which might have implications for other cases as well.

\footnotetext{
*Present address: Department of Physics, Queen's University, Kingston, Ontario K7L 3N6, Canada.
}

In the most recent survey of superallowed $0^{+} \rightarrow 0^{+}$transitions, which appeared in 2005 [1], the results for all precisely measured cases - there were 12 at that time-were statistically consistent with one another. Today, there are 13 such cases, and they still form a statistically consistent ensemble overall. However, recent precise Penning-trap measurements [6,7] of the $Q_{\mathrm{EC}}$ value for the superallowed decay of ${ }^{46} \mathrm{~V}$ have left the result for that transition more than two standard deviations away from the average of all other well-known transitions. This possible anomaly led us initially to reexamine the isospin-symmetry-breaking corrections for the ${ }^{46} \mathrm{~V}$ transition, but what we learned from that reexamination prompted us to a more general reevaluation of the corrections for other transitions as well.

Our previous shell-model calculations for ${ }^{46} \mathrm{~V}$ considered six valence nucleons occupying the $p f$-shell orbitals outside a ${ }^{40} \mathrm{Ca}$ closed shell. This model space generated reasonable energies and spins for the known states in ${ }^{46} \mathrm{Ti}$, the daughter of ${ }^{46} \mathrm{~V}$. However, an important part of the charge-dependent correction depends on the radial mismatch between the decaying proton in the parent nucleus and the resulting neutron in the daughter nucleus; but both these nucleons are bound to ${ }^{45} \mathrm{Ti}$, so the structure of that nucleus turns out to be important too. What is most striking about ${ }^{45} \mathrm{Ti}$ is that it has a $3 / 2^{+}$state at an excitation energy of only $330 \mathrm{keV}$, which is strongly populated in single-nucleon pickup reactions such as $(p, d)$ and $\left({ }^{3} \mathrm{He}, \alpha\right)$. Such low-lying $s d$-shell states can contribute to the structural parentage of the initial and final states of the superallowed transition and consequently must affect the radial mismatch between them. This indicated to us that a complete calculation of the isospin-symmetry-breaking correction for the decay of ${ }^{46} \mathrm{~V}$ should include contributions from shells deeper than the $p f$ shell.

Two questions then arose. How many deeper shells need to be included? And, if this effect is important for ${ }^{46} \mathrm{~V}$ decay, 
how many other transitions will be similarly affected? In Sec. III of this paper, we address these questions and settle on criteria for including deeper shells. Using these criteria-and incorporating more recent effective interactions that have become available since our last work-we then reevaluate the isospin-symmetry-breaking corrections for all transitions of relevance to the study of superallowed $0^{+} \rightarrow 0^{+} \beta$ decay. For the cases with $A \leqslant 38$, the changes in the corrections are very small-typically $0.03 \%$ - but for the heavier nuclei, the changes can be as large as $0.2 \%$. Most significantly, with the new calculated corrections, the result for ${ }^{46} \mathrm{~V}$ is no longer anomalous.

In Sec. IV, we incorporate recent improvements made by Marciano and Sirlin [8] to the calculation of the radiative corrections for superallowed decays; and then in Sec. V, we apply both types of corrections-isospin-symmetrybreaking and radiative-to the current experimental data for superallowed decays. The result for $V_{\mathrm{ud}}$ is changed appreciably, although it is still within the quoted uncertainties of its old value, and the CKM-unitarity sum is improved.

\section{SUPERALLOWED $\beta$ DECAY}

Superallowed Fermi $\beta$ decay between $0^{+}$states depends uniquely on the vector part of the hadronic weak interaction. When it occurs between isospin $T=1$ analog states, the conserved vector current (CVC) hypothesis indicates that the $f t$ values should be the same irrespective of the nucleus, viz.,

$$
f t=\frac{K}{G_{V}^{2}\left|M_{F}\right|^{2}}=\mathrm{const},
$$

where $K /(\hbar c)^{6}=2 \pi^{3} \hbar \ln 2 /\left(m_{e} c^{2}\right)^{5}=(8120.278 \pm 0.004) \times$ $10^{-10} \mathrm{GeV}^{-4} \mathrm{~s} ; G_{V}$ is the vector coupling constant for semileptonic weak interactions; and $M_{F}$ is the Fermi matrix element. The CVC hypothesis asserts that the vector coupling constant $G_{V}$ is a true constant and not renormalized to another value in the nuclear medium.

In practice, Eq. (1) has to be amended slightly. First, there are radiative corrections because, for example, the emitted electron may emit a bremsstrahlung photon that goes undetected in the experiment. Second, isospin is not an exact symmetry in nuclei, so the nuclear matrix element $M_{F}$ is slightly reduced from its ideal value, leading us to write

$$
\left|M_{F}\right|^{2}=\left|M_{0}\right|^{2}\left(1-\delta_{C}\right),
$$

where $M_{0}$ is the exact-symmetry value, which for $T=1$ states is $M_{0}=\sqrt{2}$. Thus, we define a "corrected" $\mathcal{F} t$ value as

$$
\mathcal{F} t \equiv f t\left(1+\delta_{R}\right)\left(1-\delta_{C}\right)=\frac{K}{2 G_{V}^{2}\left(1+\Delta_{R}^{V}\right)}=\mathrm{const},
$$

where $\delta_{C}$ is the isospin-symmetry-breaking correction, $\delta_{R}$ is the transition-dependent part of the radiative correction, and $\Delta_{R}^{V}$ is the transition-independent part. Fortunately, these corrections are all of order 1\%; but even so, to maintain an accuracy criterion of $0.1 \%$, they must be calculated with an accuracy of $10 \%$ of their central value. This is a demanding request, especially for the nuclear-structure-dependent corrections.
To separate out those terms that are dependent on nuclear structure from those that are not, we split the transitiondependent radiative correction into two terms,

$$
\delta_{R}=\delta_{R}^{\prime}+\delta_{\mathrm{NS}}
$$

of which the first, $\delta_{R}^{\prime}$, is a function only of the electron's energy and the charge of the daughter nucleus $Z$; it therefore depends on the particular nuclear decay but is independent of nuclear structure. The second term, $\delta_{\mathrm{NS}}$, like $\delta_{C}$, depends in its evaluation on the details of nuclear structure. To emphasize the different sensitivities of the correction terms, we rewrite the expression for $\mathcal{F} t$ as

$$
\mathcal{F} t \equiv f t\left(1+\delta_{R}^{\prime}\right)\left(1+\delta_{\mathrm{NS}}-\delta_{C}\right)=\frac{K}{2 G_{V}^{2}\left(1+\Delta_{R}^{V}\right)},
$$

where the first correction in brackets is independent of nuclear structure, while the second incorporates the structuredependent terms.

From Eq. (5) it can be seen that a measurement of any one superallowed transition establishes a single value for $G_{V}$; moreover, measurement of many transitions provides an excellent test of the validity of the whole analysis. Since CVC requires a unique value of $G_{V}$, all the extracted $\mathcal{F} t$ values should be identical within experimental uncertainties.

The $f t$ value that characterizes any $\beta$ transition depends on three measured quantities: the total transition energy $Q_{\mathrm{EC}}$; the half-life $t_{1 / 2}$ of the parent state; and the branching ratio $R$ for the particular transition of interest. The $Q_{\mathrm{EC}}$ value is required to determine the statistical rate function $f$, while the half-life and branching ratio combine to yield the partial half-life $t$. In 2005, we published a new survey of world data on superallowed $0^{+} \rightarrow 0^{+} \beta$ decays [1]. All previously published measurements were included, even those based on outdated calibrations if enough information was provided that they could be corrected to modern standards. In all, more than 125 independent measurements of comparable precision, spanning four decades, made the cut. In the two years since the survey was closed, another ten relevant publications have appeared [6,7,9-16], and we have now incorporated these results into our data base. Based on these data for the 13 most precisely known transitions, we obtain the $f t$ values shown on the left side of Fig. 1; then, by incorporating the corrections calculated by us in 2002 [4] and used in our 2005 survey [1], we obtain the corrected $\mathcal{F} t$ values plotted on the right side of the figure.

Obviously, the calculated corrections do a remarkable job eliminating the considerable scatter that is evident in the $f t$-value plot on the left but is absent in the corrected $\mathcal{F} t$ values shown on the right. Overall, the statistical agreement among the $\mathcal{F} t$ values is quite satisfactory, the normalized $\chi^{2}$ being 0.8 . Thus, considering that the correction terms were evaluated completely independently of these data, the consistency among the $\mathcal{F} t$ values can be taken as strong evidence that the correction terms are, in general, soundly based.

However, there is a small but noticeable deviation from the average at ${ }^{46} \mathrm{~V}$ (and possibly ${ }^{42} \mathrm{Sc}$ ), which has only been revealed by the recent Penning-trap measurements [6,7] of the transition $Q_{\mathrm{EC}}$ values. Though its statistical significance appears rather marginal in the figure, it must be remarked that 

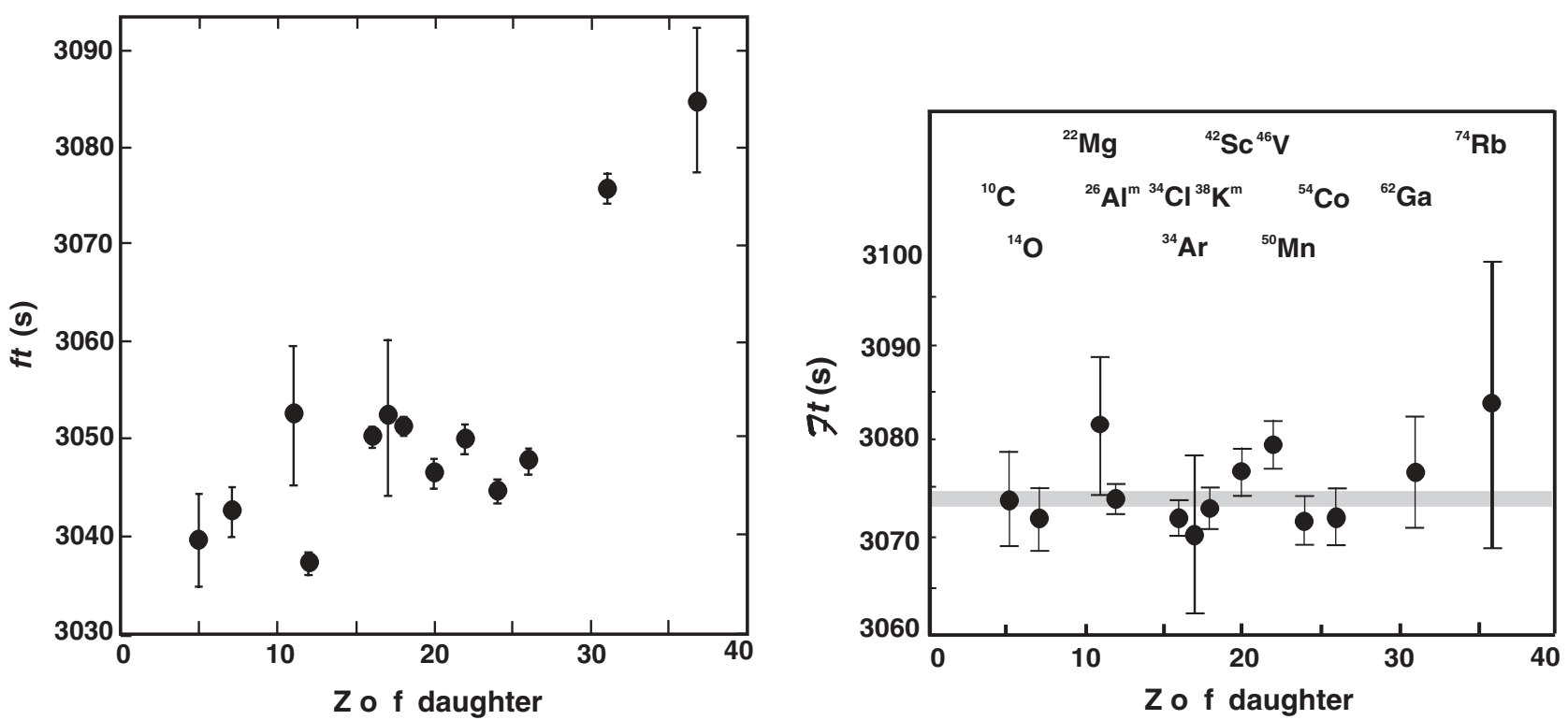

FIG. 1. Results from the 2005 survey [1] updated with more recent published results [6,7,9-16]. The uncorrected $f t$ values for the 13 best-known superallowed decays (left) are compared with the same results after corrections have been applied to obtain $\mathcal{F} t$ values (right). Here we have used the corrections calculated by us in 2002 [4], which were used in the original survey. The shaded horizontal band gives one standard deviation around the average $\mathcal{F} t$ value.

the uncertainties quoted on these $\mathcal{F} t$ values have been very conservatively determined. The measured data for each input parameter- $Q_{\mathrm{EC}}$ value, half-life, and branching ratio-were separately evaluated [1], and, if the measurements were inconsistent with one another, the weighted-average uncertainty for that parameter was increased to account for that inconsistency. In effect, for such cases, the original uncertainties quoted with the published measurements were all increased by a common "scale factor" that was large enough to restore statistical consistency among the measurements. (These scale factors are tabulated for each parameter in Ref. [1]; they range from 1 to 3.6.) This method, which is also used by the Particle Data Group [17], leads to final average values that have a high confidence level, but it does so at the cost of producing uncertainties that are in many cases larger than would result from a strict statistical average.

With this method of analysis in mind, the excursion of the ${ }^{46} \mathrm{~V} \mathcal{F} t$ value cannot be entirely ignored as a possible signal that the nuclear-structure-dependent corrections in this mass region are deficient. It certainly proved to be sufficiently provocative that we were led to the reevaluation of correction terms that is reported here.

\section{ISOSPIN-SYMMETRY-BREAKING CORRECTION $\delta_{C}$}

For weak vector interactions in hadron states, the CVC hypothesis protects the decay amplitudes from strong-interaction corrections. However, there is a caveat. The CVC hypothesis also requires the hadron state to be an exact eigenstate of $\mathrm{SU}(2)$ symmetry (isospin). In nuclei, SU(2) is always broken, albeit weakly, by Coulomb interactions between protons. There may be other charge-dependent effects as well. These influences shift the value of the hadron matrix element from its exact symmetry limit to a new value, and this shift has to be evaluated before weak-interaction physics can be probed with hadrons. In the case of superallowed $\beta$ decay, the hadron matrix element $M_{F}$ is given by Eq. (2), and it is $\delta_{C}$ that we seek to evaluate.

In the shell model for the cases of interest here, the $A$ particle wave functions representing the initial and final states for superallowed $\beta$ decay, $|i\rangle$ and $|f\rangle$, are states of angular momentum zero and isospin one. In a second quantization formulation, the Fermi matrix element is written as

$$
M_{F}=\left\langle f\left|\tau_{+}\right| i\right\rangle=\sum_{\alpha, \beta}\left\langle f\left|a_{\alpha}^{\dagger} a_{\beta}\right| i\right\rangle\left\langle\alpha\left|\tau_{+}\right| \beta\right\rangle,
$$

where the operator for Fermi $\beta$ decay is the isospin ladder operator, $a_{\alpha}^{\dagger}$ creates a neutron in quantum state $\alpha$, and $a_{\beta}$ annihilates a proton in quantum state $\beta$. The single-particle matrix element $\left\langle\alpha\left|\tau_{+}\right| \beta\right\rangle$ is just a radial integral

$$
\left\langle\alpha\left|\tau_{+}\right| \beta\right\rangle=\delta_{\alpha, \beta} \int_{0}^{\infty} R_{\alpha}^{n}(r) R_{\beta}^{p}(r) r^{2} d r \equiv \delta_{\alpha, \beta} r_{\alpha} .
$$

If the proton and neutron radial functions $R_{\alpha}^{n}(r)$ and $R_{\beta}^{p}(r)$ are identical, then the radial integral reduces to the normalization integral and has the value $r_{\alpha}=1$.

Now we introduce into Eq. (6) a complete set of states for the $(A-1)$-particle system, $|\pi\rangle$, by writing

$$
M_{F}=\sum_{\pi, \alpha}\left\langle f\left|a_{\alpha}^{\dagger}\right| \pi\right\rangle\left\langle\pi\left|a_{\alpha}\right| i\right\rangle r_{\alpha}^{\pi} .
$$

This is the essence of our model: we have allowed the radial integral to depend on the parentage expansion. Thus, we have added an additional label to $r_{\alpha}$ and now write $r_{\alpha}^{\pi}$.

If isospin is an exact symmetry, then the matrix elements of the creation and annihilation operators are related by Hermiticity, $\left\langle\pi\left|a_{\alpha}\right| i\right\rangle=\left\langle f\left|a_{\alpha}^{\dagger}\right| \pi\right\rangle^{*}$. With that requirement and 
with the radial integrals set to unity, the symmetry-limit matrix element is

$$
M_{0}=\sum_{\pi, \alpha}\left|\left\langle f\left|a_{\alpha}^{\dagger}\right| \pi\right\rangle\right|^{2} .
$$

Thus we see that the breakdown of isospin symmetry can enter the evaluation of $M_{F}$ in one of two ways: either the matrix elements of $a_{\alpha}$ and $a_{\alpha}^{\dagger}$ are not related by Hermiticity, or the radial integrals are not unity. Since each effect is small, we can, to first order, write the isospin-symmetry-breaking correction as the sum of two terms

$$
\delta_{C}=\delta_{C 1}+\delta_{C 2}
$$

where in evaluating $\delta_{C 1}$, all radial integrals are set to unity but the matrix elements are not assumed to be related by Hermiticity; while in evaluating $\delta_{C 2}$, it is assumed that $\left\langle\pi\left|a_{\alpha}\right| i\right\rangle=\left\langle f\left|a_{\alpha}^{\dagger}\right| \pi\right\rangle^{*}$, but the radial integrals are allowed to differ from unity. Past calculations $[4,5]$ have indicated the radial overlap correction, $\delta_{C 2}$, is the larger of the two corrections, so we will study this first.

\section{A. Radial overlap correction $\delta_{C 2}$}

\section{Strategy for calculation}

For the $\delta_{C 2}$ calculation, the Fermi matrix element is

$$
\begin{aligned}
M_{F} & =\sum_{\pi, \alpha}\left|\left\langle f\left|a_{\alpha}^{\dagger}\right| \pi\right\rangle\right|^{2} r_{\alpha}^{\pi} \\
& =\sum_{\pi, \alpha}\left|\left\langle f\left|a_{\alpha}^{\dagger}\right| \pi\right\rangle\right|^{2}-\sum_{\pi, \alpha}\left|\left\langle f\left|a_{\alpha}^{\dagger}\right| \pi\right\rangle\right|^{2}\left(1-r_{\alpha}^{\pi}\right) \\
& =M_{0}\left(1-\frac{1}{M_{0}} \sum_{\pi, \alpha}\left|\left\langle f\left|a_{\alpha}^{\dagger}\right| \pi\right\rangle\right|^{2} \Omega_{\alpha}^{\pi}\right),
\end{aligned}
$$

where $M_{0}$ is the exact-symmetry value, Eq. (9), and $\Omega_{\alpha}^{\pi}$ has been introduced as a radial-mismatch factor

$$
\Omega_{\alpha}^{\pi}=\left(1-r_{\alpha}^{\pi}\right) .
$$

Recalling that $\delta_{C 2}$ is defined as $\left|M_{F}\right|^{2}=\left|M_{0}\right|^{2}\left(1-\delta_{C 2}\right)$, we obtain

$$
\delta_{C 2} \simeq \frac{2}{M_{0}} \sum_{\pi, \alpha}\left|\left\langle f\left|a_{\alpha}^{\dagger}\right| \pi\right\rangle\right|^{2} \Omega_{\alpha}^{\pi}
$$

to first order in small quantities. A large contribution to $\delta_{C 2}$ therefore requires a large spectroscopic amplitude and a significant departure of the radial integral from unity.

There is an opportunity here to take guidance from experiment. The square of each spectroscopic amplitude, $\left|\left\langle f\left|a_{\alpha}^{\dagger}\right| \pi\right\rangle\right|^{2}$, is related to the spectroscopic factor measured in neutron pickup direct reactions. The exact relation, after inserting the isospin angular momentum couplings, is

$$
\delta_{C 2} \simeq \sum_{\pi, \alpha} \frac{T_{f}\left(T_{f}+1\right)+\frac{3}{4}-T_{\pi}\left(T_{\pi}+1\right)}{T_{f}\left(T_{f}+1\right)} S_{\alpha, T_{f}}^{T_{\pi}} \Omega_{\alpha}^{\pi},
$$

where $S_{\alpha, T_{f}}^{T_{\pi}}$ is the spectroscopic factor for pickup of a neutron in quantum state $\alpha$ from an $A$-particle state of isospin $T_{f}$ to an $(A-1)$-particle state of isospin $T_{\pi}$. On setting $T_{f}=1$ and separately identifying sums to the isospin-lesser states with $T_{\pi}=\frac{1}{2}$, denoted $\pi^{<}$, and the isospin-greater states with $T_{\pi}=\frac{3}{2}$, denoted $\pi^{>}$, we obtain a very revealing formula:

$$
\delta_{C 2} \simeq \sum_{\pi^{<}, \alpha} S_{\alpha}^{<} \Omega_{\alpha}^{<}-\frac{1}{2} \sum_{\pi^{>}, \alpha} S_{\alpha}^{>} \Omega_{\alpha}^{>} .
$$

This equation provides the key to the strategy we will use in calculating $\delta_{C 2}$. It demonstrates that there is a cancellation between the contributions of the isospin-lesser states and the isospin-greater states. Moreover, if the orbital $\alpha$ were completely full in the initial $A$-particle wave function, then the Macfarlane and French sum rules [18] for spectroscopic factors would require $\sum_{\pi^{<}} S_{\alpha}^{<}=\frac{1}{2} \sum_{\pi^{>}} S_{\alpha}^{>}$and the cancellation in Eq. (15) would be very strong. In fact, the cancellation would be complete if $\Omega_{\alpha}^{<}=\Omega_{\alpha}^{>}$. As we will discuss further in the next section, this cancellation is not in general complete because the radial-mismatch factors for isospin-lesser states are larger than those for isospin-greater states. Even so, cancellation is always significant, and it becomes most complete when closed-shell orbitals are involved. Furthermore, the more deeply bound the closed-shell orbital, the greater the energy spread in the spectroscopic strength and the more complete the cancellation. Thus, although the dominant contributions to $\delta_{C 2}$ come from unfilled orbitals, we conclude that closed-shell orbitals must play a role, albeit one that decreases in importance as the orbitals become more deeply bound.

Based on these observations, our strategy is to use experiment to guide us in determining which closed-shell orbitals are important enough to include. Ideally, of course, one would take the spectroscopic factors determined from experiment and insert them into Eq. (15), but, especially where delicate cancellations are involved, the reliability of (40-year-old) experimental spectroscopic factors is certainly not up to the task. Our strategy then is to use the shell model to calculate the spectroscopic amplitudes in Eq. (13) but to limit the sum over orbitals $\alpha$ just to those for which large spectroscopic factors have been observed in neutron pickup reactions.

We illustrate the strategy for the case of ${ }^{46} \mathrm{~V}$. The spectroscopic factors for neutron pickup from ${ }^{46} \mathrm{Ti}$ have been measured in the $\left({ }^{3} \mathrm{He}, \alpha\right)$ reaction by Borlin [19]. He identified 16 states in ${ }^{45} \mathrm{Ti}$, and in Table I we record the six states with the largest spectroscopic factors, i.e., $S>0.5$. We note that the errors on the experimental spectroscopic factors are quite large, and in two cases the quoted $S_{\alpha}$ values (column 4) exceed the Macfarlane-French (M-F) sum rule [18] for pure configurations (column 6). Thus we do not use the experimental spectroscopic factors explicitly, but take them as a guide for which orbitals should be included in the shell-model calculation. In the case of ${ }^{46} \mathrm{~V}$ decay, they tell us that orbitals $f_{7 / 2}, d_{3 / 2}$, and $s_{1 / 2}$ should be included. In column 5 of Table I we give a typical value for the radial mismatch factor $\Omega_{\alpha}^{\pi}$ for the given orbital $\alpha$ and isospin $T_{\pi}$. Column 7 gives the contribution to $\delta_{C 2}$ from this $\alpha$ and isospin $T_{\pi}$ if the Macfarlane-French sum rule is used for the spectroscopic factor, while columns 8 and 9 show the results of a detailed shell-model calculation. The results from the Macfarlane-French sum rules and the shell-model calculation are remarkably similar. The summed $\delta_{C 2}$ for the shell-model calculation (the sum of all entries in 
TABLE I. Illustration of the strategy used in calculating $\delta_{C 2}$ for ${ }^{46} \mathrm{~V}$. The measured spectroscopic factors from the ${ }^{46} \mathrm{Ti}\left({ }^{3} \mathrm{He}, \alpha\right)^{45} \mathrm{Ti}$ reaction [19] are shown for the states where they are largest. Two calculations are then given for each state's contribution to $\delta_{C 2}$ : the first assumes that the total Macfarlane-French (M-F) sum rule is exhausted in each state, while the second gives the result of a complete shell-model calculation. Both methods give remarkably similar results.

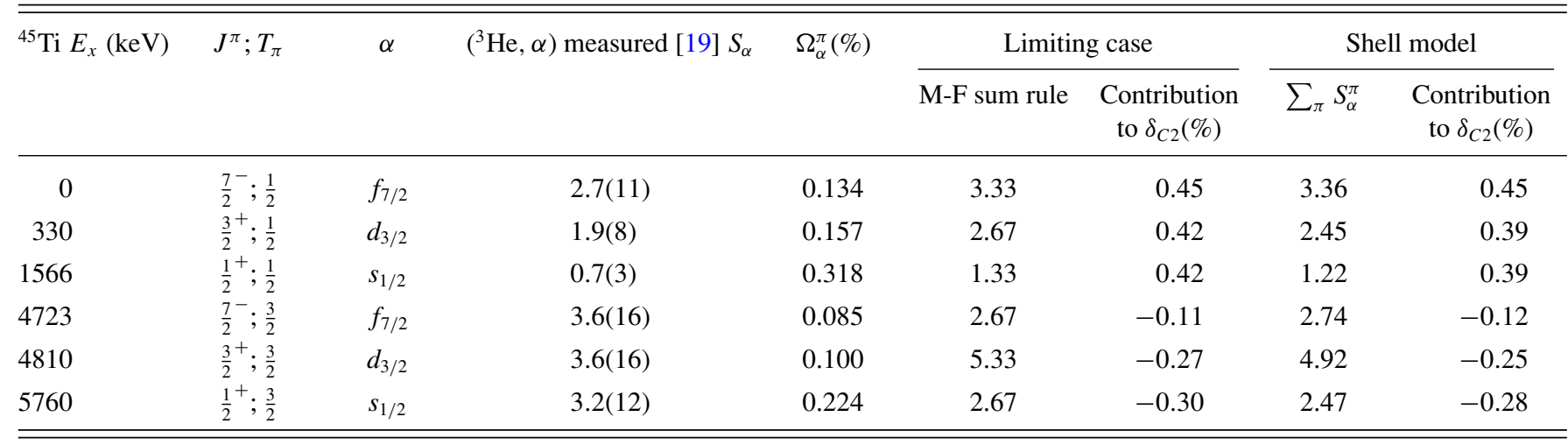

column 9) is $0.58 \%$, nearly a factor of 2 larger than our previous calculated value, which was published in 2002 [4].

The difference between our calculations arises as follows: In 2002 our shell-model calculations for ${ }^{46} \mathrm{~V}$ were based on the model space $(f p)^{6}$, with six valence nucleons occupying the $p f$-shell orbitals. In fact, only the $f_{7 / 2}$ orbital contributed importantly to the $\delta_{C 2}$ calculation, so the result was $\delta_{C 2}=$ $0.45-0.12=0.33 \%$ (see the two rows for the $f_{7 / 2}$ orbital in Table I). Absent from this 2002 calculation was any contribution from the core orbitals, $d_{3 / 2}$ and $s_{1 / 2}$. In our present calculations, these orbitals are included, with the $d_{3 / 2}$ orbital contributing $0.14 \%$ to $\delta_{C 2}$, and the $s_{1 / 2}$ contributing $0.11 \%$.

But why stop there? Why not include the $d_{5 / 2}$ and possibly the $p$-shell orbitals in the computation? We considered this question carefully, even though a detailed calculation was precluded by the size of the extended shell-model configuration space, which exceeded our numerical capabilities. As a limiting case, we took just one $d_{5 / 2}$ state with $T_{\pi}=\frac{1}{2}$ and one with $T_{\pi}=\frac{3}{2}$, each carrying the Macfarlane-French sum rule for its spectroscopic strength. Then, with an estimate of its location in the ${ }^{45} \mathrm{Ti}$ spectrum, we could obtain the contribution to $\delta_{C 2}$ from these two deep-hole states. Since the neutron pickup experiment saw little or no evidence for such core states, it is likely that the actual spectroscopic strength is highly fragmented and not simply confined to a pair of states. In that case, the cancellation between isospin-lesser and isospin-greater states becomes more complete, and their contribution to $\delta_{C 2}$ is reduced. Thus, with our simple model almost certainly giving an over-estimate of the effect, we found that the possible contribution of deep-hole states to $\delta_{C 2}$ is less than our assigned uncertainties.

With this approach, we are now in a position to revise our earlier results [4] to include the effects of previously ignored core orbitals. Again using measured spectroscopic factors from neutron pickup reactions, we determined that changes were required for the $A=22$ and 26 cases, in which $p$-shell holes must contribute in addition to the original $s d$-shell configurations; similarly, $s d$-shell holes were required in addition to the $p f$-shell particles for $A=46,50$, and 54 . For $A=62,66,70$, and 74 in the upper $p f$-shell, there are no experimental neutron pickup reaction measurements to guide us. Our previously published calculations for these nuclei were based on $\left(p_{3 / 2}, f_{5 / 2}, p_{1 / 2}\right)^{n}$ model spaces using ${ }^{56} \mathrm{Ni}$ as a closed-shell core. It seemed prudent now for these cases at least to include the $f_{7 / 2}$ orbital in the calculation of $\delta_{C 2}$, and we have made this change. In the cases with $A=18$ and 42, we had previously included some contribution from deeper shells; we did not need to make any changes in the former but did add the $s_{1 / 2}$ and $d_{5 / 2}$ shells to the latter. No additional orbitals were required for the cases with $A=10,14,30,34$, and 38 .

\section{Radial-mismatch factor $\Omega_{\alpha}^{\pi}$}

In considering the radial integrals, we benefit from a very strong constraint: the asymptotic forms of all radial functions must match the measured separation energies $S_{p}$ and $S_{n}$, where $S_{p}$ is the proton separation energy in the decaying nucleus and $S_{n}$ the neutron separation energy in the daughter nucleus. The basic ingredients of these separation energies are well known and can be found in any atomic mass table. It is the size of the difference between $S_{p}$ and $S_{n}$ and the presence or absence of nodes in the radial wave functions that are the principal factors in determining the magnitude of $\Omega_{\alpha}^{\pi}$.

Our calculations of this mismatch factor follows the same path as that described in our earlier works $[4,20]$. We use a Saxon-Woods potential defined for a nucleus of mass $A$ and charge $Z+1$ as

$V(r)=-V_{0} f(r)-V_{s} g(r) \mathbf{l} \cdot \boldsymbol{\sigma}+V_{C}(r)-V_{g} g(r)-V_{h} h(r)$,

where

$$
\begin{aligned}
f(r)= & \left\{1+\exp \left(\frac{r-R}{a}\right)\right\}^{-1}, \\
g(r)= & \left(\frac{\hbar}{m_{\pi} c}\right)^{2} \frac{1}{a_{s} r} \exp \left(\frac{r-R_{s}}{a_{s}}\right) \\
& \times\left\{1+\exp \left(\frac{r-R_{s}}{a_{s}}\right)\right\}^{-2},
\end{aligned}
$$




$$
\begin{aligned}
h(r) & =a^{2}\left(\frac{d f}{d r}\right)^{2}, \\
V_{C}(r) & =Z e^{2} / r, \quad \text { for } r \geqslant R_{c}, \\
& =\frac{Z e^{2}}{2 R_{c}}\left(3-\frac{r^{2}}{R_{c}^{2}}\right), \quad \text { for } \quad r<R_{c},
\end{aligned}
$$

with $R=r_{0}(A-1)^{1 / 3}$ and $R_{s}=r_{s}(A-1)^{1 / 3}$. The first three terms in Eq. (16) are the central, spin-orbit, and Coulomb terms, respectively. The fourth and fifth terms are additional surface terms whose role we discuss shortly.

Most of the parameters were fixed at standard values, $V_{s}=7 \mathrm{MeV}, r_{s}=1.1 \mathrm{fm}$, and $a=a_{s}=0.65 \mathrm{fm}$. The radius of the Coulomb potential was determined from the charge mean square radius $\left\langle r^{2}\right\rangle_{\mathrm{ch}}^{1 / 2}$ of the decaying nucleus as determined from elastic electron scattering; see Eqs. (21) and (22) in Ref. [4]. The well radius $r_{0}$ was similarly fixed, by requiring that the charge density constructed from the square of the proton wave functions bound in the well should also match the charge mean square radius. Initially, with $V_{g}$ and $V_{h}$ set to zero, the well depth $V_{0}$ was adjusted so that the binding energy of the least-bound orbital matched the experimental separation energy.

From the shell-model calculation, we obtained the $A$ particle wave functions, $|i\rangle$ and $|f\rangle$, expanded into products of $(A-1)$-particle wave functions $|\pi\rangle$ and single-particle functions $|\alpha\rangle$. In Eq. (8) and the discussion that followed it, we noted that the radial integral should depend on the separation energies relative to the $(A-1)$ state, $|\pi\rangle$. We ultimately allowed this to happen, but initially we calculated the value of $\delta_{C 2}$ under the assumption that the proton and neutron radial functions, $R^{p}(r)$ and $R^{n}(r)$, have asymptotic forms for all $\alpha$ that are fixed at the separation energies $S_{p}$ and $S_{n}$ to the ground state of the $(A-1)$ nucleus. In this case, the sums over $\pi$ can be done analytically, and the computed value of $\delta_{C 2}$ becomes independent of the shell-model effective interaction. This result, which we label $\delta_{C 2}^{\mathrm{I}}$, can be simply expressed with the help of Eqs. (9) and (13):

$$
\delta_{C 2}^{\mathrm{I}} \simeq 2 \Omega_{\alpha_{g}} \text {. }
$$

Here $\alpha_{g}$ is the shell-model orbital of the transferred neutron in the pickup reaction from the $A$-particle state $|f\rangle$ to the ground state of the $(A-1)$-particle nucleus.

We next removed our simplifying assumption and evaluated the radial integrals with eigenfunctions of the Saxon-Woods potential whose well depth was adjusted so that each eigenfunction matched the separation energy of the $(A-1)$ state to which it corresponds, $|\pi\rangle$. For an $(A-1)$ state at excitation energy $E_{x}$, the corresponding separation energies are $S_{p}+E_{x}$ and $S_{n}+E_{x}$. We label these results $\delta_{C 2}^{\mathrm{II}}$ and note that the values now depend on the spectroscopic amplitudes, and hence on the shell-model effective interaction, but not strongly.

So far, we have ignored the two surface terms in Eq. (17) by setting $V_{g}=0$ and $V_{h}=0$. It can be argued, however, that the central part of the potential, which in principle should be determined from some Hartree-Fock procedure, should not be continually adjusted. Instead, any adjustments made to match separation energies should be to the surface part of the potential rather than to the depth of the well. Thus, we also calculated
$\delta_{C 2}$ by fixing $V_{0}$ separately for protons and neutrons to match the ground-state parent separation energies $S_{p}$ and $S_{n}$, and then adjusting the strength of the surface term $V_{g}$ (keeping $V_{h}=0$ ) so that the asymptotic forms matched the separation energies $S_{p}+E_{x}$ and $S_{n}+E_{x}$. These results are labeled $\delta_{C 2}^{\mathrm{III}}$.

Finally, our fourth method of calculation was the same as the third, except that it was the second surface term $V_{h}$ that was adjusted to match separation energies, keeping $V_{g}=0$. This second term $h(r)$ is even more strongly peaked in the surface than $g(r)$. These results are labeled $\delta_{C 2}^{\mathrm{IV}}$.

On average, the method III values of $\delta_{C 2}$ are about $2 \%$ lower than the method II values; and method IV values are about $7 \%$ lower than the method II values for orbitals without any radial nodes. For orbitals with one or more nodes, there is more of the radial wave function in the surface region and methods III and IV produce greater reductions.

\section{Shell-model calculations}

We now present our results for $\delta_{C 2}$ based on the extensions of the shell-model spaces mentioned at the end of Sec. III A1. In addition to adding the core orbitals mentioned there, however, in some cases we have also been able to make use of more recent effective interactions that have become available since our last work. Specifically, we used the following interactions in the various mass regions of interest: In the $p$ shell, we used the Cohen-Kurath interactions [21] and the more recent PWBT interaction of Warburton and Brown [22]. In the $s, d$ shell, besides the universal interaction of Wildenthal [23], we employed two new versions, USD-A and USD-B, of Brown and Richter [24]. In the $p f$ shell, we used the KB3 interaction of Kuo-Brown [25] as modified by Poves and Zuker [26], the FPMI3 interaction of Richter and Brown [27], and the more recent GXPF1 interaction of Honma et al. [28,29]. For cross-shell interactions between the major shells, we used the interaction of Millener and Kurath [30]. Note that in many cases we found it necessary to introduce some truncations in the original model space in order to keep the calculations tractible.

We made calculations for all 20 superallowed transitions considered in our earlier work [1,4], and for each we calculated $\delta_{C 2}$ in the four methods, I-IV, described in Sec. III A2 and with the several interactions listed in the previous paragraph. In Table II, we record only one sample result for $\delta_{C 2}^{\mathrm{I}}, \delta_{C 2}^{\mathrm{II}}, \delta_{C 2}^{\mathrm{III}}$, and $\delta_{C 2}^{\mathrm{IV}}$ for each nucleus listed. However, our "adopted $\delta_{C 2}$ ", values result from our assessment of all multiple-parentage calculations made for each decay, not just those shown in the previous three columns. The uncertainty assigned to each adopted value reflects the uncertainty in the radius of the Saxon-Woods potential (resulting from an uncertainty in the nuclear rms radius to which it is adjusted), the spread of results obtained with different shell-model interactions, and the spread of results obtained with the different procedures labeled II, III, and IV in the table.

\section{B. Isospin-mixing correction $\delta_{C 1}$}

The second (and smaller) contribution to $\delta_{C}$ is the isospinmixing correction $\delta_{C 1}$. For its evaluation, the radial integrals 
TABLE II. Calculations of $\delta_{C 2}$ with Saxon-Woods radial functions, without parentage expansions $\left(\delta_{C 2}^{I}\right)$ and with parentage expansions $\left(\delta_{C 2}^{\mathrm{II}}, \delta_{C 2}^{\mathrm{III}}\right.$, and $\left.\delta_{C 2}^{\mathrm{IV}}\right)$. Note that only one sample result is shown in each case for $\delta_{C 2}^{I}, \delta_{C 2}^{\mathrm{II}}, \delta_{C 2}^{\mathrm{III}}$ and $\delta_{C 2}^{\mathrm{IV}}$, while the adopted $\delta_{C 2}$ value in column 7 reflects the results from all multiple-parentage calculations for that case; see text.

\begin{tabular}{|c|c|c|c|c|c|c|}
\hline \multirow{2}{*}{$\begin{array}{l}\text { Parent } \\
\text { nucleus }\end{array}$} & \multirow{2}{*}{$\begin{array}{c}2002 \delta_{C 2}(\%) \\
\text { Ref. [4] }\end{array}$} & \multicolumn{5}{|c|}{ This work } \\
\hline & & $\delta_{C 2}^{\mathrm{I}}(\%)$ & $\delta_{C 2}^{\mathrm{II}}(\%)$ & $\delta_{C 2}^{\mathrm{III}}(\%)$ & $\delta_{C 2}^{\mathrm{IV}}(\%)$ & $\delta_{C 2}(\%)$ adopted \\
\hline \multicolumn{7}{|l|}{$T_{z}=-1:$} \\
\hline${ }^{10} \mathrm{C}$ & $0.170(15)$ & 0.132 & 0.163 & 0.165 & 0.163 & $0.165(15)$ \\
\hline${ }^{14} \mathrm{O}$ & $0.270(15)$ & 0.217 & 0.274 & 0.271 & 0.271 & $0.275(15)$ \\
\hline${ }^{18} \mathrm{Ne}$ & $0.390(10)$ & 0.251 & 0.386 & 0.387 & 0.382 & $0.410(25)$ \\
\hline${ }^{22} \mathrm{Mg}$ & $0.255(10)$ & 0.207 & 0.366 & 0.382 & 0.375 & $0.370(20)$ \\
\hline${ }^{26} \mathrm{Si}$ & $0.330(10)$ & 0.223 & 0.421 & 0.407 & 0.392 & $0.405(25)$ \\
\hline${ }^{30} \mathrm{~S}$ & $0.740(20)$ & 0.812 & 0.714 & 0.710 & 0.713 & $0.700(20)$ \\
\hline${ }^{34} \mathrm{Ar}$ & $0.610(40)$ & 0.351 & 0.680 & 0.639 & 0.579 & $0.635(55)$ \\
\hline${ }^{38} \mathrm{Ca}$ & $0.710(50)$ & 0.402 & 0.840 & 0.784 & 0.702 & $0.745(70)$ \\
\hline${ }^{42} \mathrm{Ti}$ & $0.555(40)$ & 0.359 & 0.881 & 0.849 & 0.780 & $0.835(75)$ \\
\hline \multicolumn{7}{|l|}{$T_{z}=0$} \\
\hline${ }^{26} \mathrm{Al}^{m}$ & $0.230(10)$ & 0.156 & 0.292 & 0.280 & 0.271 & $0.280(15)$ \\
\hline${ }^{34} \mathrm{Cl}$ & $0.530(30)$ & 0.312 & 0.583 & 0.561 & 0.498 & $0.550(45)$ \\
\hline${ }^{38} \mathrm{~K}^{m}$ & $0.520(40)$ & 0.299 & 0.623 & 0.575 & 0.522 & $0.550(55)$ \\
\hline${ }^{42} \mathrm{Sc}$ & $0.430(30)$ & 0.278 & 0.681 & 0.648 & 0.606 & $0.645(55)$ \\
\hline${ }^{46} \mathrm{~V}$ & $0.330(25)$ & 0.273 & 0.587 & 0.543 & 0.506 & $0.545(55)$ \\
\hline${ }^{50} \mathrm{Mn}$ & $0.450(30)$ & 0.315 & 0.638 & 0.598 & 0.594 & $0.610(50)$ \\
\hline${ }^{54} \mathrm{Co}$ & $0.570(40)$ & 0.376 & 0.760 & 0.688 & 0.706 & $0.720(60)$ \\
\hline${ }^{62} \mathrm{Ga}$ & $1.05(15)$ & 1.31 & 1.22 & 1.19 & 1.14 & $1.20(20)$ \\
\hline${ }^{66} \mathrm{As}$ & $1.15(15)$ & 1.32 & 1.41 & 1.34 & 1.24 & $1.35(40)$ \\
\hline${ }^{70} \mathrm{Br}$ & $1.00(20)$ & 1.43 & 1.41 & 1.31 & 1.10 & $1.25(25)$ \\
\hline${ }^{74} \mathrm{Rb}$ & $1.30(40)$ & 1.68 & 1.60 & 1.47 & 1.12 & $1.50(30)$ \\
\hline
\end{tabular}

are all set to unity, but the spectroscopic amplitudes in Eq. (8) are not required to satisfy Hermiticity. Calculations of this correction turn out to be very sensitive to the details of the shell-model computation. This would be a very unfortunate property if we were not able to adopt certain strategies that act to reduce the model dependence considerably.

There are three ways in which we incorporated charge dependence in our shell-model calculation. First, the singleparticle energies of the proton orbits were shifted relative to those of the neutrons. The amount of shift was determined from the spectrum of single-particle states in the closed-shell-plusproton versus the closed-shell-plus-neutron nucleus, where the closed shell was taken to be the nucleus used as a closed-shell core in the shell-model calculation. We took these single-particle shifts from experiment and did not adjust them.

Second, we added a two-body Coulomb interaction among the valence protons and adjusted its strength so that the measured $b$ coefficient of the isobaric multiplet mass equation (IMME) was exactly reproduced. Third, we introduced a charge-dependent nuclear interaction by increasing all the $T=1$ proton-neutron matrix elements by about $2 \%$ relative to the neutron-neutron matrix elements. The precise amount of this increment was determined by requiring agreement with the measured $c$ coefficient of the IMME. This strategy of constraining the charge dependence in the effective interaction by requiring it to reproduce the coefficients of the IMME was adopted from the work of Ormand and Brown $[31,32]$.
Experimental data were used in one more way to constrain our calculations. If isospin were an exact symmetry, then the parent $0^{+}(T=1)$ state would decay exclusively to its analog state in the daughter nucleus, and $\beta$ transitions to all other $0^{+}$ states in the daughter would be strictly forbidden. But, with isospin symmetry broken, weak transitions (with branching ratios measured in parts per million) can occur to these other $0^{+}$states. In this case, we write the Fermi matrix element squared to the $n$th non-analog $0^{+}$state as

$$
\left|M_{F}^{n}\right|^{2}=2 \delta_{C 1}^{n},
$$

and the reduction in the analog transition Fermi matrix element squared as

$$
\left|M_{F}\right|^{2}=2\left(1-\delta_{C 1}\right),
$$

neglecting, in this context, the contribution of $\delta_{C 2}$. If all the $0^{+}$ states of a given model space had the same $T=1$ isospin designation, then the effect of isospin-symmetry-breaking terms in the Hamiltonian would be to deplete the analogtransition strength by an amount that is exactly matched by the sum of the strengths to the non-analog states; i.e.,

$$
\delta_{C 1} \simeq \sum_{n} \delta_{C 1}^{n} .
$$

In practice, with large shell-model calculations the $0^{+}$states in the model space will include some states whose isospin 
TABLE III. Shell-model calculations of the isospin-mixing correction, $\delta_{C 1}$.

\begin{tabular}{|c|c|c|c|c|c|c|c|c|}
\hline \multirow{3}{*}{$\begin{array}{l}\text { Parent } \\
\text { nucleus }\end{array}$} & \multirow{2}{*}{\multicolumn{2}{|c|}{$\begin{array}{r}\text { Measured IMME } \\
\text { coefficients [33] }\end{array}$}} & \multirow{3}{*}{$\begin{array}{c}2002 \delta_{C 1}(\%) \\
\text { Ref. [4] }\end{array}$} & \multicolumn{5}{|c|}{ This work } \\
\hline & & & & \multirow{2}{*}{$E_{x}\left(0^{+}\right) \operatorname{expt}$} & \multirow{2}{*}{$\begin{array}{c}E_{x}\left(0^{+}\right) \\
\mathrm{SM}\end{array}$} & \multirow{2}{*}{$\begin{array}{c}\delta_{C 1}(\%) \\
\text { unscaled }\end{array}$} & \multirow{2}{*}{$\begin{array}{c}\delta_{C 1}(\%) \\
\text { scaled }\end{array}$} & \multirow{2}{*}{$\begin{array}{c}\delta_{C 1}(\%) \\
\text { adopted }\end{array}$} \\
\hline & $b(\mathrm{keV})$ & $c(\mathrm{keV})$ & & & & & & \\
\hline \multicolumn{9}{|l|}{$T_{z}=-1:$} \\
\hline${ }^{10} \mathrm{C}$ & -1.546 & 0.362 & $0.010(10)$ & 6.18 & 9.24 & 0.005 & 0.011 & $0.010(10)$ \\
\hline${ }^{14} \mathrm{O}$ & -2.493 & 0.337 & $0.050(20)$ & 6.59 & 6.64 & 0.049 & 0.050 & $0.055(20)$ \\
\hline${ }^{18} \mathrm{Ne}$ & $-3.045(1)$ & $0.347(1)$ & $0.230(30)$ & 3.71 & 4.07 & 0.116 & 0.140 & $0.155(30)$ \\
\hline${ }^{22} \mathrm{Mg}$ & $-3.814(1)$ & $0.315(1)$ & $0.010(10)$ & 6.24 & 6.21 & 0.010 & 0.010 & $0.010(10)$ \\
\hline${ }^{26} \mathrm{Si}$ & $-4.535(2)$ & $0.302(2)$ & $0.040(10)$ & 3.59 & 3.86 & 0.022 & 0.026 & $0.030(10)$ \\
\hline${ }^{30} \mathrm{~S}$ & $-5.185(2)$ & $0.275(2)$ & $0.195(30)$ & 3.79 & 3.80 & 0.137 & 0.138 & $0.155(20)$ \\
\hline${ }^{34} \mathrm{Ar}$ & $-5.777(2)$ & $0.286(2)$ & $0.030(10)$ & 3.92 & 3.97 & 0.023 & 0.023 & $0.030(10)$ \\
\hline${ }^{38} \mathrm{Ca}$ & $-6.328(3)$ & $0.284(3)$ & $0.020(10)$ & 3.38 & 3.21 & 0.026 & 0.023 & $0.020(10)$ \\
\hline${ }^{42} \mathrm{Ti}$ & $-6.712(3)$ & $0.287(3)$ & $0.220(100)$ & 1.84 & 3.16 & 0.038 & 0.114 & $0.100(20)$ \\
\hline \multicolumn{9}{|l|}{$T_{z}=0$} \\
\hline${ }^{26} \mathrm{Al}^{m}$ & $-4.535(2)$ & $0.302(2)$ & $0.040(10)$ & 3.59 & 3.86 & 0.025 & 0.028 & $0.030(10)$ \\
\hline${ }^{34} \mathrm{Cl}$ & $-5.777(2)$ & $0.286(2)$ & $0.105(20)$ & 3.92 & 3.97 & 0.091 & 0.093 & $0.100(10)$ \\
\hline${ }^{38} \mathrm{~K}^{m}$ & $-6.328(3)$ & $0.284(3)$ & $0.100(20)$ & 3.38 & 3.21 & 0.099 & 0.089 & $0.105(20)$ \\
\hline${ }^{42} \mathrm{Sc}$ & $-6.712(3)$ & $0.287(3)$ & $0.060(30)$ & $3.30^{\mathrm{a}}$ & 5.05 & 0.007 & 0.017 & $0.020(10)$ \\
\hline${ }^{46} \mathrm{~V}$ & $-7.327(10)$ & $0.276(11)$ & $0.095(20)$ & $3.57^{\mathrm{a}}$ & 4.86 & 0.040 & 0.075 & $0.075(30)$ \\
\hline${ }^{50} \mathrm{Mn}$ & $-7.892(30)$ & $0.259(30)$ & $0.055(20)$ & 3.69 & 3.62 & 0.057 & 0.054 & $0.045(20)$ \\
\hline${ }^{54} \mathrm{Co}$ & $-8.519(25)$ & $0.276(25)$ & $0.040(15)$ & 2.56 & 2.26 & 0.058 & 0.045 & $0.050(30)$ \\
\hline${ }^{62} \mathrm{Ga}$ & $-9.463(70)$ & $0.265(25)^{\mathrm{b}}$ & $0.330(40)$ & 2.33 & 2.32 & 0.221 & 0.219 & $0.275(55)$ \\
\hline${ }^{66} \mathrm{As}$ & $-9.95(15)$ & $0.262(25)^{\mathrm{b}}$ & $0.250(40)$ & $2.17^{\mathrm{c}}$ & 1.89 & 0.210 & 0.159 & $0.205(45)$ \\
\hline${ }^{70} \mathrm{Br}$ & $-10.48(23)$ & $0.260(25)^{\mathrm{b}}$ & $0.350(40)$ & 2.01 & 2.05 & 0.332 & 0.346 & $0.350(40)$ \\
\hline${ }^{74} \mathrm{Rb}$ & $-10.82(25)$ & $0.258(25)^{\mathrm{b}}$ & $0.130(60)$ & 0.508 & 0.523 & 0.122 & 0.129 & $0.130(60)^{\mathrm{d}}$ \\
\hline
\end{tabular}

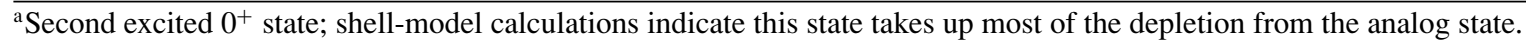

${ }^{b}$ Estimated: extrapolated from a fit to $c$ coefficients in $0^{+}$states in $A=4 n+2$ nuclei, $10 \leqslant A \leqslant 58$; the data were taken from Ref. [33].

${ }^{c}$ Estimated: value is the average of the excitation energy of the $0^{+}$states in ${ }^{62} \mathrm{Zn}$ and ${ }^{70} \mathrm{Se}$.

${ }^{\mathrm{d}}$ No new calculations were performed for ${ }^{74} \mathrm{Rb}$.

designation is not $T=1$; and then Eq. (21) is not exactly correct. Nevertheless, it remains approximately true.

Significantly, in many cases the bulk of the analog state depletion shows up in a single excited $0^{+}$state, usually (but not always) the first excited one. This allows us once again to use experiment to constrain and refine our calculation. In the limit of only two-state mixing, perturbation theory would indicate that

$$
\delta_{C 1} \propto \frac{1}{(\Delta E)^{2}},
$$

where $\Delta E$ is the energy separation of the analog and nonanalog $0^{+}$states. Again, this is not an exact result, but it does highlight the importance of the shell-model Hamiltonian producing a good quality spectrum of $0^{+}$states with, in particular, the first excited non-analog $0^{+}$state calculated to have an excitation energy close to its experimental value. ${ }^{1}$ This is not always possible to achieve in the shell model, especially

\footnotetext{
${ }^{1}$ In a few cases, the state calculated to have the largest chargedependent admixture was the second excited $0^{+}$state. In these cases, we optimized the agreement between theory and experiment for the excitation energy of that state.
}

near closed shells where excited $0^{+}$states tend to exhibit strong deformations. We used two strategies to bring the calculation into line with experimental information. Our first was to adjust the centroids of the shell-model Hamiltonian matrix elements specifically to get the excited $0^{+}$state at about the right energy. Our second was to scale our calculated $\delta_{C 1}$ value by a factor of $(\Delta E)_{\text {theo }}^{2} /(\Delta E)_{\text {expt }}^{2}$, the ratio of the square of the excitation energy of the first excited $0^{+}$state in the model calculation to that known experimentally.

We list in Table III the experimental values [33] of the IMME coefficients $b$ and $c$ and the known excitation energy $E_{x}\left(0^{+}\right)$of the first (or second) excited $0^{+}$state in the daughter nuclei. As explained, all our shell-model calculations were adjusted to reproduce exactly the values of $b$ and $c$, and to match, as closely as possible the excitation energy of the excited $0^{+}$state. We compensated for any remaining discrepancies between the calculated and experimental values of $E_{x}\left(0^{+}\right)$by scaling the results for $\delta_{C 1}$. As in Table II, we give (in columns 6-8) the results from one sample calculation for each nucleus. Then in column nine we present adopted $\delta_{C 1}$ values that result from our assessment of the results of all calculations made for each decay, not just the ones shown in columns 6-8; the uncertainties were chosen to encompass the spread in the results from those calculations and to include the 
TABLE IV. Shell-model calculations of $\delta_{C 1}^{1}$ for Fermi decay to the first excited $0^{+}$state; see Eq. (19). The results are compared with experimental measurements where they are known. All values are expressed in $\%$.

\begin{tabular}{lccccc}
\hline \hline \multirow{2}{*}{$\begin{array}{l}\text { Parent } \\
\text { nucleus }\end{array}$} & $\begin{array}{c}2002 \\
\text { value [4] }\end{array}$ & \multicolumn{3}{c}{ This work } & \multirow{2}{*}{ Expt } \\
\cline { 3 - 5 } & & Unscaled & Scaled & Adopted & \\
\hline$T_{z}=0:$ & & & & & \\
${ }^{38} \mathrm{~K}^{m}$ & $0.090(30)$ & 0.068 & 0.062 & $0.085(30)$ & $<0.28^{\mathrm{a}}$ \\
${ }^{42} \mathrm{Sc}$ & $0.020(20)$ & 0.007 & 0.027 & $0.015(15)$ & $0.040(9)^{\mathrm{b}}$ \\
${ }^{46} \mathrm{~V}$ & $0.035(15)$ & 0.008 & 0.024 & $0.025(20)$ & $0.053(5)^{\mathrm{a}}$ \\
${ }^{50} \mathrm{Mn}$ & $0.045(20)$ & 0.049 & 0.047 & $0.040(20)$ & $<0.016^{\mathrm{a}}$ \\
${ }^{54} \mathrm{Co}$ & $0.040(20)$ & 0.049 & 0.038 & $0.050(20)$ & $0.035(5)^{\mathrm{a}}$ \\
${ }^{62} \mathrm{Ga}$ & $0.085(20)$ & 0.160 & 0.159 & $0.120(40)$ & $\leqslant 0.040(15)^{\mathrm{c}}$ \\
${ }^{66} \mathrm{As}$ & $0.020(20)$ & 0.110 & 0.087 & $0.050(30)$ & \\
${ }^{70} \mathrm{Br}$ & $0.070(20)$ & 0.226 & 0.235 & $0.150(80)$ & \\
${ }^{74} \mathrm{Rb}$ & $0.050(30)$ & 0.045 & 0.047 & $0.050(30)^{\mathrm{d}}$ & $\leqslant 0.075^{\mathrm{e}}$ \\
\hline \hline
\end{tabular}

${ }^{\mathrm{a}}$ From Hagberg et al. (1994) [34].

${ }^{\text {b}}$ From Daehnick and Rosa (1985) [35] averaged with earlier results.

${ }^{\mathrm{c}}$ From Hyland et al. (2006) [15].

${ }^{\mathrm{d}}$ No new calculations were performed for ${ }^{74} \mathrm{Rb}$.

${ }^{\mathrm{e}}$ From Piechaczek et al. (2003) [36].

uncertainty in the IMME $b$ and $c$ coefficients. For comparison, in column 4 we list the values we adopted for $\delta_{C 1}$ in 2002 [4]. Our strategies have remained unchanged, but here we have additionally used some more recent shell-model effective interactions as listed in Sec. III A3. In nearly all cases, the new values of $\delta_{C 1}$ agree with the old values within their stated uncertainties.

For the heavier nuclei, there are experimental data on Fermi transitions to the non-analog excited $0^{+}$states. The measured branching ratios [15,34-36] have been converted to $\delta_{C 1}^{1}$ values, via Eq. (19), and listed in Table IV. Again, for each nucleus, we list just one representative calculation and our adopted value. The assigned error reflects both the spread among the different calculations and the uncertainties in the IMME coefficients. Our 2002 adopted values [4] are also listed. For nuclei $38 \leqslant A \leqslant 54$, with the possible exception of ${ }^{50} \mathrm{Mn}$, the agreement between theory and experiment is entirely satisfactory. But in the upper $p f$ shell, the calculated value for ${ }^{62} \mathrm{Ga}$ is three times larger than measured in recent experiments [15]. Shell-model calculations in this region are complicated by the massive size of the Hamiltonian matrices. To keep our calculations tractible, we kept the $f_{7 / 2}$ shell closed in these cases, but there is considerable evidence [29] that this could be a poor assumption.

\section{THE RADIATIVE CORRECTION}

\section{A. Prior to 1990}

Conventionally, the radiative correction has been separated into two parts, one that contains the nucleus-dependent terms, called the "outer" radiative correction, and one that is independent of the nucleus, the "inner" radiative correction. Principally due to the work of Marciano and Sirlin (for example, Refs. [37-39]), the radiative correction applied to the uncorrected $\beta$-decay rate $\Gamma_{\beta}^{0}$ was expressed as

$$
\begin{aligned}
\Gamma_{\beta} & =\Gamma_{\beta}^{0}\left(1+\delta_{R}^{\prime}\right)\left(1+\Delta_{R}^{V}\right) \\
\delta_{R}^{\prime} & =\frac{\alpha}{2 \pi}\left[\bar{g}\left(E_{m}\right)+\delta_{2}+\delta_{3}\right] \\
\stackrel{\operatorname{large} E_{m}}{\longrightarrow} & \frac{\alpha}{2 \pi}\left[3 \ln \left(\frac{m_{p}}{2 E_{m}}\right)+\frac{81}{10}-\frac{4 \pi^{2}}{3}+\delta_{2}+\delta_{3}\right], \\
\Delta_{R}^{V} & =\frac{\alpha}{2 \pi}\left[3 \ln \frac{m_{W}}{m_{p}}+\ln \frac{m_{W}}{m_{A}}+2 C-4 \ln \frac{m_{W}}{m_{Z}}+\mathcal{A}_{g}\right] \\
& =\frac{\alpha}{2 \pi}\left[4 \ln \frac{m_{Z}}{m_{p}}+\ln \frac{m_{p}}{m_{A}}+2 C+\mathcal{A}_{g}\right],
\end{aligned}
$$

where $E_{m}$ is the maximum electron energy in $\beta$ decay, and $m_{W}, m_{p}, m_{Z}$ are the masses of the $W$ boson, proton, and $Z$ boson. The separation into outer and inner terms is accommodated in $\delta_{R}^{\prime}$ and $\Delta_{R}^{V}$, respectively.

In the outer correction $\delta_{R}^{\prime}$, the order- $\alpha$ term contains the function $\bar{g}\left(E_{m}\right)$ : it is the average over the $\beta$ energy spectrum of the function $g\left(E, E_{m}\right)$, which was defined by Sirlin (see Eq. (20b) of Ref. [37]) and is not reproduced here. Its large- $E_{m}$ limit is shown in Eq. (24), indicating that the expression is dominated by the logarithm $\ln \left(m_{p} /\left(2 E_{m}\right)\right)$. The last two terms in the outer correction, $\delta_{2}$ and $\delta_{3}$, represent corrections to order $Z \alpha^{2}$ and $Z^{2} \alpha^{3}$, respectively. The origin of the $\bar{g}\left(E_{m}\right)$ termtogether with that of the leading term in the inner radiative correction, $3 \ln \left(m_{W} / m_{p}\right)$-is the $\gamma W$-box and bremsstrahlung diagrams, which are taken together to remove the divergence as the photon energy goes to zero. Both $\delta_{2}$ and $\delta_{3}$ also come from a standard QED calculation of the $\gamma W$-box and bremsstrahlung graphs [40,41], but in their case, the electron was allowed to interact with the Coulomb field of the nucleus. Care was taken not to double count with the Fermi function. The calculation was complete to order $Z \alpha^{2}$ but only estimated in order $Z^{2} \alpha^{3}$. 
In the inner correction $\Delta_{R}^{V}$, the second and third terms, $\ln \left(m_{W} / m_{A}\right)+2 C$, like the first term, also represent a $\gamma W$-box graph, but this time it involves an axial-vector weak interaction. The evaluation of this graph can be divided into two energy regimes: the high-energy (or short-distance) part given by the logarithm, and the low-energy (or long-distance) part denoted by $2 C$. The parameter $m_{A}$, referred to as the low-energy cutoff, divides these two energy regimes. Marciano and Sirlin [38] allowed it to take on a range of values, $400 \leqslant m_{A} \leqslant 1600 \mathrm{MeV}$ (revised slightly by Sirlin [42] to be $m_{a_{1}} / 2 \leqslant m_{A} \leqslant 2 m_{a_{1}}$, with $m_{a_{1}}$ being the $A_{1}$-vector-meson mass). The low-energy component, $2 C$, was approximated by its Born contribution

$$
C \rightarrow C_{\text {Born }}=3 g_{A}(0.266)\left(\mu_{p}+\mu_{n}\right)=0.885,
$$

where $g_{A}=1.26$ is the axial vector coupling constant accepted at the time, and $\left(\mu_{p}+\mu_{n}\right)=0.88$ is the nucleon isoscalar magnetic moment. The factor 0.266 is the value of the loop integral that was rendered finite by the use of dipole form factors for the nucleon electromagnetic, $\gamma N$, and axial-vector, $W N$, vertices. The fourth term in Eq. (25), with the logarithm $\ln \left(m_{W} / m_{Z}\right)$, arises from $Z W$-box graphs; while the last term, $\mathcal{A}_{g}$, represents a small perturbative QCD correction that was evaluated by Marciano and Sirlin [39] to be $\mathcal{A}_{g}=-0.34$.

The value of the outer radiative correction as defined in Eq. (24), ranges from $1.39 \%$ to $1.65 \%$ for the known superallowed emitters (see Ref. [4]). Following Sirlin [40], the assigned uncertainties are set equal to $(\alpha / 2 \pi) \delta_{3}$ as an estimate of the error made in stopping the calculation at that order. The value of the inner radiative correction as obtained from Eq. (26) with $C$ from Eq. (27) is [39,42]

$$
\Delta_{R}^{V}(\text { old })=2.40(8) \% \text {. }
$$

These results provide the essential foundation of the radiation corrections still used today. However, a number of improvements have been introduced in the intervening 17 years.

\section{B. A nuclear-structure-dependent term}

The low-energy part of the $\gamma W$-box diagram for an axial-vector weak interaction, denoted $2 C$, was approximated by its Born contribution in Eq. (27), and was evaluated on a single nucleon. However, in a finite nucleus with many nucleons present, Jaus and Rasche [43] observed that the two hadronic-interaction vertices, $\gamma N$ and $W N$, do not have to be with the same nucleon. Thus, in finite nuclei, there can be two types of contributions: those in which $\gamma N$ and $W N$ vertices are with the same nucleon and those in which they are not. The evaluation of the former terms yields expressions [44] that are proportional to $\tau_{+}$, the isospin ladder operator, and so are also proportional to the Fermi $\beta$-decay operator. Therefore, they produce a universal correction-the same

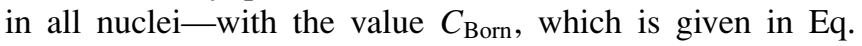
(27). The remaining terms, those in which the interactions are with different nucleons, must be evaluated with two-body operators that depend on the nuclear structure of the states involved. Thus, the expression for $C$ given in Eq. (27) must be replaced by

$$
C=C_{\mathrm{Born}}+C_{\mathrm{NS}}
$$

where $C_{\mathrm{NS}}$ comprises the nuclear-structure-dependent terms. Calculations of $C_{\mathrm{NS}}$ were first made in 1992 [44,45].

A further modification was introduced in 1994 [46]. In calculations of $C_{\text {Born }}$ that had been made up to that time, the axial-vector and electromagnetic coupling constants, $g_{A}$ and $\left(\mu_{p}+\mu_{n}\right)$-see Eq. (27)—had been given their free-nucleon values. Yet there is ample evidence in nuclear physics that coupling constants for spin-flip processes are quenched in the nuclear medium, with the amount of quenching varying from nucleus to nucleus. Thus, one should really be replacing $C_{\text {Born }}^{\text {free }}$, the value obtained with free-nucleon coupling constants, with $C_{\text {Born }}^{\text {quened }}$. However, to separate the nucleus-dependent and nucleus-independent parts of the latter, we write

$$
\begin{aligned}
C_{\mathrm{Born}}^{\text {quenched }} & =q C_{\text {Born }}^{\text {free }} \\
& =C_{\text {Born }}^{\text {free }}+(q-1) C_{\text {Born }}^{\text {free }},
\end{aligned}
$$

where $q$ is the factor by which the product of the weak and electromagnetic coupling constants is reduced in the medium relative to its free-nucleon value.

The first term in Eq. (30), which remains universal, is retained in the inner radiative correction, replacing $C$ in Eq. (25). The second term becomes part of a separate nuclear-structure-dependent radiative correction, $\delta_{\mathrm{NS}}$, which also includes $C_{\mathrm{NS}}^{\text {quenched }}$, the value of $C_{\mathrm{NS}}$ recalculated with quenched operators. This correction is written as

$$
\delta_{\mathrm{NS}}=\frac{\alpha}{\pi}\left[C_{\mathrm{NS}}^{\text {quenched }}+(q-1) C_{\mathrm{Born}}^{\text {free }}\right],
$$

and is incorporated with the other nuclear-structure-dependent correction term, $\delta_{C}$; see Eq. (5). Calculated values of $\delta_{\mathrm{NS}}$ [4] range from $-0.360 \%$ to $+0.030 \%$, each generally being smaller in magnitude than the corresponding value of $\delta_{C}$.

We will return to $\delta_{\mathrm{NS}}$ in Sec. IV E.

\section{Improvements to $\Delta_{R}^{V}$}

In 2005, Czarnecki, Marciano, and Sirlin [47] revisited the $\mathcal{O}\left(\alpha^{2}\right)$ correction for neutron $\beta$ decay. They began by trivially updating the value of $C_{\text {Born }}^{\text {free }}$ to reflect the current value of the axial-vector coupling constant, $g_{A}=1.27$, to get

$$
C_{\text {Born }}^{\text {free }}=0.891 \text {, }
$$

which replaces the value given in Eq. (27).

They then went on to reevaluate $\Delta_{R}^{V}$, focusing particularly on the leading log corrections. Using an established renormalization group summation [39] for the leading short-distance logs, $S\left(m_{p}, m_{Z}\right)$, they extended the method to the lower energy region between $2 E_{m}$ and $m_{p}$ to obtain $L\left(2 E_{m}, m_{p}\right)$. This resulted in the replacements

$$
\begin{aligned}
1+\frac{2 \alpha}{\pi} \ln \frac{m_{Z}}{m_{p}} & \rightarrow S\left(m_{p}, m_{Z}\right)=1.02248, \\
1+\frac{3 \alpha}{2 \pi} \ln \frac{m_{p}}{2 E_{m}} & \rightarrow L\left(2 E_{m}, m_{p}\right),
\end{aligned}
$$


where

$$
L\left(2 E_{m}, m_{p}\right)=1.026725\left[1-\frac{2 \alpha\left(m_{e}\right)}{3 \pi} \ln \frac{2 E_{m}}{m_{e}}\right]^{9 / 4} .
$$

The complete radiative correction, $\mathrm{RC}$, including order $Z \alpha^{2}$ and $Z^{2} \alpha^{3}$ terms, could then be written [47] as

$$
\begin{aligned}
1+\mathrm{RC}= & \left\{1+\frac{\alpha}{2 \pi}\left[\bar{g}\left(E_{m}\right)-3 \ln \frac{m_{p}}{2 E_{m}}\right]\right\} \\
& \times\left\{L\left(2 E_{m}, m_{p}\right)+\frac{\alpha}{2 \pi}\left[2 C_{\mathrm{Born}}^{\text {free }}+\delta_{2}+\delta_{3}\right]\right\} \\
& \times\left\{S\left(m_{p}, m_{Z}\right)+\frac{\alpha\left(m_{p}\right)}{2 \pi}\left[\ln \frac{m_{p}}{m_{A}}+\mathcal{A}_{g}\right]+\mathrm{NLL}\right\},
\end{aligned}
$$

where NLL is a next-to-leading log correction that Czarnecki et al. estimate to be NLL $=-0.0001$. The coefficient $\alpha(m)$ is a running QED coupling constant whose value at $m=m_{p}$ is $1 / 133.986$ and at $m=m_{e}$ is $1 / 137.089$ [47].

This new result can still be organized to preserve the separation of nucleus-dependent and nucleus-independent components. The separation we hereby adopt is

$$
\begin{aligned}
1+\delta_{R}^{\prime}= & \left\{1+\frac{\alpha}{2 \pi}\left[\bar{g}\left(E_{m}\right)-3 \ln \frac{m_{p}}{2 E_{m}}\right]\right\} \\
& \times\left\{L\left(2 E_{m}, m_{p}\right)+\frac{\alpha}{2 \pi}\left[\delta_{2}+\delta_{3}\right]\right\}, \\
1+\Delta_{R}^{V}= & \frac{1+\mathrm{RC}}{1+\delta_{R}^{\prime}} \cong S\left(m_{p}, m_{Z}\right)+\frac{\alpha}{\pi} C_{\text {Born }}^{\text {free }} \\
& +\frac{\alpha\left(m_{p}\right)}{2 \pi}\left[\ln \frac{m_{p}}{m_{A}}+\mathcal{A}_{g}\right]+\text { NLL. }
\end{aligned}
$$

We will use this separation here and in our future work on superallowed $\beta$ decay. It results in a small change to the values of $\delta_{R}^{\prime}$ and $\Delta_{R}^{V}$ that we used in our recent review [1].

\section{Reduced uncertainty for $\Delta_{R}^{V}$}

In Sec. IV A, we explained that the terms $\ln \left(m_{W} / m_{A}\right)+2 C$ in Eq. (25) arose from the $\gamma W$-box graph for an axial-vector weak interaction. These two terms came from splitting the evaluation of this graph into two energy regimes. The division between the two regimes was chosen to be $m_{A}=1.2 \mathrm{GeV}$ [42], roughly the mass of the $A_{1}$ resonance, and its range of uncertainty was taken to be from $m_{A} / 2$ to $2 m_{A}$. This adhoc range determination actually produced the largest single contributor to the uncertainty in the CKM matrix element $V_{\text {ud }}$.

To reduce the hadronic uncertainty in the radiative correction, Marciano and Sirlin [8] looked again at the $\gamma W$-box graph for an axial-vector weak interaction. This time they split it into three energy regimes rather than two, and, where possible, they drew on independent information to control their results:

(i) Short distances, $(1.5 \mathrm{GeV})^{2} \leqslant Q^{2}<\infty$ : This is a domain where QCD corrections remain perturbative. Marciano and Sirlin added higher-order terms, noting that these terms are identical (in the chiral limit) to
QCD corrections to the Bjorken sum rule for polarized electroproduction and can therefore be obtained from well-studied calculations for that process.

(ii) Intermediate distances, $(0.823 \mathrm{GeV})^{2} \leqslant Q^{2}<$ $(1.5 \mathrm{GeV})^{2}$ : In this region, they used an interpolation function between low and high energies, motivated by vector-meson and axial-vector-meson dominance. By limiting the number of terms to three, they had sufficient matching conditions to determine the coefficients uniquely.

(iii) Long distances, $0 \leqslant Q^{2} \leqslant(0.823 \mathrm{GeV})^{2}$ : Integrating the long-distance amplitudes up to $Q^{2}=(0.823 \mathrm{GeV})^{2}$, where the integrand matches smoothly to the interpolation function, they obtained a smaller value for $C_{\mathrm{Born}}^{\mathrm{free}}$,

$$
C_{\text {Born }}^{\text {free }}=0.829 \text {, }
$$

than given in Eq. (32). However this smaller value is caused entirely by the reduction in the effective upper limit to the loop integration and is almost completely compensated for by the consequently higher values obtained for the graph in the other energy regimes.

In the end, Marciano and Sirlin [8] find that the net effect of this reevaluation of the $\gamma W$-box axial graph is a very small reduction in the radiative correction of $1.4 \times 10^{-4}$. More important than this reduction, the new method provides a more systematic estimate of the hadronic uncertainties. Allowing for a $\pm 10 \%$ uncertainty for the $C_{\text {Born }}^{\text {free }}$ correction in Eq. (39), a $\pm 100 \%$ uncertainty for the interpolator contribution in the intermediate region, and \pm 0.0001 uncertainty from neglected higher-order effects, Marciano and Sirlin [8] find the total uncertainty in the radiative correction to be \pm 0.00038 . This corresponds to more than a factor of 2 reduction in the loop uncertainty for hadronic effects [cf. Eq. (28)].

\section{E. New values for $\delta_{R}^{\prime}, \Delta_{R}^{V}$, and $\delta_{\mathrm{NS}}$}

We maintain the traditional separation of the radiative correction into a nucleus-dependent outer correction and a nucleus-independent inner correction; see Eqs. (37) and (38). This means that the outer correction $\delta_{R}^{\prime}$ is slightly redefined and is now written as

$$
\delta_{R}^{\prime}=\frac{\alpha}{2 \pi}\left[\bar{g}\left(E_{m}\right)+\delta_{2}+\delta_{3}+\delta_{\alpha^{2}}\right],
$$

where the new term, $\delta_{\alpha^{2}}$, simply represents the difference between the definition of $\delta_{R}^{\prime}$ given in Eq. (37) and that given in Eq. (24). It is the leading-log extrapolation of the logarithm $\ln \left(m_{p} / 2 E_{m}\right)$, which is contained in the function $\bar{g}\left(E_{m}\right)$. Values of $\delta_{\alpha^{2}}$ and the redefined $\delta_{R}^{\prime}$ are given in Table $\mathrm{V}$ for all the superallowed transitions of interest.

The new inner correction is defined by Eq. (38), with $C_{\text {Born }}^{\text {free }}$ taken from Eq. (39). With its uncertainty obtained from Marciano and Sirlin [8], the result is

$$
\Delta_{R}^{V}=(2.361 \pm 0.038) \% \text {. }
$$

It is important to note that with the reevaluation of $C_{\text {Born }}^{\text {free }}$, there is a consequent change in the nuclear-structure- 
TABLE V. Calculated transition-dependent radiative correction $\delta_{R}^{\prime}$ in percent units, and the component contributions. In our previous works (e.g., Ref. [4]) $\delta_{R}^{\prime}$ was defined as the sum of the contents of columns 2-4; this result is given in column 5 and labeled "Former $\delta_{R}^{\prime}$." As explained in the text, we have now redefined $\delta_{R}^{\prime}$ to include the additional term in column 6; the new values for $\delta_{R}^{\prime}$ are given in the last column.

\begin{tabular}{lcccccc}
\hline \hline $\begin{array}{l}\text { Parent } \\
\text { nucleus }\end{array}$ & $\frac{\alpha}{2 \pi} \bar{g}\left(E_{m}\right)$ & $\frac{\alpha}{2 \pi} \delta_{2}$ & $\frac{\alpha}{2 \pi} \delta_{3}$ & $\begin{array}{c}\text { Former } \\
\delta_{R}^{\prime}\end{array}$ & $\frac{\alpha}{2 \pi} \delta_{\alpha^{2}}$ & $\begin{array}{c}\text { Redefined } \\
\delta_{R}^{\prime}\end{array}$ \\
\hline$T_{z}=-1:$ & & & & & & \\
${ }^{10} \mathrm{C}$ & 1.468 & 0.180 & 0.004 & 1.652 & 0.027 & $1.679(4)$ \\
${ }^{14} \mathrm{O}$ & 1.286 & 0.226 & 0.008 & 1.520 & 0.023 & $1.543(8)$ \\
${ }^{18} \mathrm{Ne}$ & 1.204 & 0.268 & 0.012 & 1.484 & 0.022 & $1.506(12)$ \\
${ }^{22} \mathrm{Mg}$ & 1.122 & 0.307 & 0.017 & 1.446 & 0.020 & $1.466(17)$ \\
${ }^{26} \mathrm{Si}$ & 1.055 & 0.342 & 0.023 & 1.420 & 0.019 & $1.439(23)$ \\
${ }^{30} \mathrm{~S}$ & 1.005 & 0.371 & 0.029 & 1.405 & 0.018 & $1.423(29)$ \\
${ }^{34} \mathrm{Ar}$ & 0.963 & 0.396 & 0.035 & 1.395 & 0.017 & $1.412(35)$ \\
${ }^{38} \mathrm{Ca}$ & 0.929 & 0.426 & 0.042 & 1.397 & 0.017 & $1.414(42)$ \\
${ }^{42} \mathrm{Ti}$ & 0.906 & 0.456 & 0.050 & 1.412 & 0.016 & $1.428(50)$ \\
${ }_{z}=0:$ & & & & & & \\
${ }^{26} \mathrm{Al}{ }^{m}$ & 1.110 & 0.328 & 0.020 & 1.458 & 0.020 & $1.478(20)$ \\
${ }^{34} \mathrm{Cl}$ & 1.002 & 0.390 & 0.032 & 1.425 & 0.018 & $1.443(32)$ \\
${ }^{38} \mathrm{~K}{ }^{m}$ & 0.964 & 0.420 & 0.039 & 1.423 & 0.017 & $1.440(39)$ \\
${ }^{42} \mathrm{Sc}$ & 0.939 & 0.451 & 0.047 & 1.436 & 0.017 & $1.453(47)$ \\
${ }^{46} \mathrm{~V}$ & 0.903 & 0.472 & 0.054 & 1.429 & 0.016 & $1.445(54)$ \\
${ }^{50} \mathrm{Mn}$ & 0.873 & 0.494 & 0.062 & 1.430 & 0.015 & $1.445(62)$ \\
${ }^{54} \mathrm{Co}$ & 0.844 & 0.513 & 0.071 & 1.428 & 0.015 & $1.443(71)$ \\
${ }^{62} \mathrm{Ga}$ & 0.805 & 0.553 & 0.087 & 1.445 & 0.014 & $1.459(87)$ \\
${ }^{66} \mathrm{As}$ & 0.791 & 0.570 & 0.095 & 1.456 & 0.014 & $1.470(95)$ \\
${ }^{70} \mathrm{Br}$ & 0.776 & 0.591 & 0.105 & 1.473 & 0.013 & $1.49(11)$ \\
${ }^{74} \mathrm{Rb}$ & 0.761 & 0.609 & 0.115 & 1.485 & 0.013 & $1.50(12)$ \\
\hline \hline & & & & & & \\
\hline \hline
\end{tabular}

dependent correction $\delta_{\mathrm{NS}}$ given in Eq. (31). Fortunately, the change is very small, being

$$
\begin{aligned}
(q-1)\left(C_{\mathrm{Born}}^{\mathrm{new}}-C_{\mathrm{Born}}^{\mathrm{old}}\right)\left(\frac{\alpha}{\pi}\right) & \simeq-0.3(-0.062) 2.3 \times 10^{-3} \\
& \simeq 0.004 \% .
\end{aligned}
$$

In addition to making this change, we have also taken the opportunity to reevaluate $C_{\mathrm{NS}}$ using the more recently available shell-model effective interactions described in Sec. III A3. Our revised $\delta_{\mathrm{NS}}$ values are listed in Table VI. As in Tables II and III, we give (in columns 3 and 4) the results from one sample calculation for each nucleus. Then in column 5 we present adopted $\delta_{\mathrm{NS}}$ values that result from our assesment of all calculations made for each decay, not just the ones shown in columns 3 and 4; the uncertainties were chosen to encompass the spread in the results from those calculations. For comparison, in column 2 we list the values we adopted for $\delta_{\mathrm{NS}}$ in 2002 [4]. In all cases, the new values agree with the old ones within the quoted uncertainties.

\section{V. $\mathcal{F} t$ VALUES, $V_{\text {ud }}$, AND CKM UNITARITY}

We have calculated improved results for the correction terms $\delta_{C 1}$ (see Table III), $\delta_{C 2}$ (Table II), and $\delta_{\mathrm{NS}}$ (Table VI); and, based on the work of Marciano and Sirlin, we have presented revised values for $\delta_{R}^{\prime}$ (Table V) and $\Delta_{R}^{V}$ [Eq. (41)].
TABLE VI. Calculated nuclear-structure-dependent radiative

\begin{tabular}{|c|c|c|c|c|}
\hline \multirow{2}{*}{$\begin{array}{l}\text { Parent } \\
\text { nucleus }\end{array}$} & \multirow{2}{*}{$\begin{array}{c}2002 \\
\delta_{\mathrm{NS}}(\%) \\
\text { Ref. [4] }\end{array}$} & \multicolumn{3}{|c|}{ This work } \\
\hline & & $C_{\mathrm{NS}}^{\text {quenched }}$ & $(q-1) C_{\mathrm{Born}}^{\mathrm{free}}$ & $\begin{array}{c}\delta_{\mathrm{NS}}(\%) \\
\text { adopted }\end{array}$ \\
\hline
\end{tabular}
correction, $\delta_{\mathrm{NS}}$, in percent units, and the component contributions.

\begin{tabular}{lrrrr}
\hline$T_{z}=-1:$ & & & & \\
${ }^{10} \mathrm{C}$ & $-0.360(35)$ & -1.318 & -0.176 & $-0.345(35)$ \\
${ }^{14} \mathrm{O}$ & $-0.250(50)$ & -0.844 & -0.208 & $-0.245(50)$ \\
${ }^{18} \mathrm{Ne}$ & $-0.290(35)$ & -1.051 & -0.198 & $-0.290(35)$ \\
${ }^{22} \mathrm{Mg}$ & $-0.240(20)$ & -0.750 & -0.213 & $-0.225(20)$ \\
${ }^{26} \mathrm{Si}$ & $-0.230(20)$ & -0.705 & -0.227 & $-0.215(20)$ \\
${ }^{30} \mathrm{~S}$ & $-0.190(15)$ & -0.557 & -0.242 & $-0.185(15)$ \\
${ }^{34} \mathrm{Ar}$ & $-0.185(15)$ & -0.520 & -0.257 & $-0.180(15)$ \\
${ }^{38} \mathrm{Ca}$ & $-0.180(15)$ & -0.475 & -0.271 & $-0.175(15)$ \\
${ }^{42} \mathrm{Ti}$ & $-0.240(20)$ & -0.765 & -0.241 & $-0.235(20)$ \\
${ }_{Z}=0:$ & & & & \\
${ }^{26} \mathrm{Al}{ }^{m}$ & $0.009(20)$ & 0.242 & -0.227 & $0.005(20)$ \\
${ }^{34} \mathrm{Cl}$ & $-0.085(15)$ & -0.118 & -0.257 & $-0.085(15)$ \\
${ }^{38} \mathrm{~K}^{m}$ & $-0.100(15)$ & -0.158 & -0.271 & $-0.100(15)$ \\
${ }^{42} \mathrm{Sc}$ & $0.030(20)$ & 0.391 & -0.241 & $0.035(20)$ \\
${ }^{46} \mathrm{~V}$ & $-0.040(7)$ & 0.093 & -0.248 & $-0.035(10)$ \\
${ }^{50} \mathrm{Mn}$ & $-0.042(7)$ & 0.084 & -0.254 & $-0.040(10)$ \\
${ }^{54} \mathrm{Co}$ & $-0.029(7)$ & 0.112 & -0.261 & $-0.035(10)$ \\
${ }^{62} \mathrm{Ga}$ & $-0.040(20)$ & 0.087 & -0.272 & $-0.045(20)$ \\
${ }^{66} \mathrm{As}$ & $-0.050(20)$ & 0.010 & -0.278 & $-0.060(20)$ \\
${ }^{70} \mathrm{Br}$ & $-0.060(20)$ & -0.085 & -0.283 & $-0.085(25)$ \\
${ }^{74} \mathrm{Rb}$ & $-0.065(20)$ & -0.026 & -0.288 & $-0.075(30)$ \\
\hline \hline
\end{tabular}

We are now in a position to extract corrected $\mathcal{F} t$ values from the current world data for superallowed $0^{+} \rightarrow 0^{+}$transitions.

We use the same data set as that described in Sec. II: it represents an interim update of our 2005 complete survey [1] and includes ten additional published measurements [6,7,916]. Results are given in Table VII for the 13 superallowed transitions whose $f t$ values are known to a precision of $0.3 \%$ or better. The $\mathcal{F} t$ values given in column 6 were obtained from the data in the preceding columns through the application of Eq. (5). The corrected $\mathcal{F} t$ values are also plotted in Fig. 2.

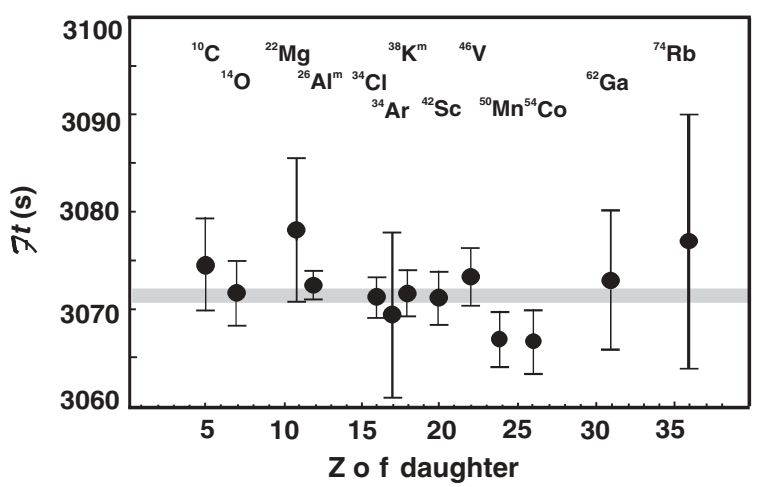

FIG. 2. Results for the new corrected $\mathcal{F} t$ values (from Table VII) for the 13 best-known superallowed decays. The corresponding uncorrected $f t$ values appear in the left panel of Fig. 1. The shaded horizontal band gives one standard deviation around the average $\overline{\mathcal{F} t}$ value. 
TABLE VII. Corrected $\mathcal{F} t$ values for the 13 best-known superallowed decays, obtained with the new correction terms presented in this work. The experimental $f t$ values were taken from results in our 2005 survey [1] updated with more recent published data [6,7,9-16]. The average $\overline{\mathcal{F} t}$ value and the normalized $\chi^{2}$ of the fit to a constant appear at the bottom.

\begin{tabular}{lccccc}
\hline \hline Parent nucleus & $f t(\mathrm{~s})$ & $\delta_{R}^{\prime}(\%)$ & $\delta_{\mathrm{NS}}(\%)$ & $\delta_{C}(\%)$ & $\mathcal{F} t(\mathrm{~s})$ \\
\hline$T_{z}=-1:$ & & & & \\
${ }^{10} \mathrm{C}$ & $3039.5(47)$ & $1.679(4)$ & $-0.345(35)$ & $0.175(18)$ & $3074.5(49)$ \\
${ }^{14} \mathrm{O}$ & $3042.5(27)$ & $1.543(8)$ & $-0.245(50)$ & $0.330(25)$ & $3071.6(33)$ \\
${ }^{22} \mathrm{Mg}$ & $3052.2(72)$ & $1.466(17)$ & $-0.225(20)$ & $0.380(22)$ & $3078.3(74)$ \\
${ }^{34} \mathrm{Ar}$ & $3052.5(82)$ & $1.412(35)$ & $-0.180(15)$ & $0.665(56)$ & $3069.4(85)$ \\
$T_{z}=0:$ & $3037.0(11)$ & $1.478(20)$ & $0.005(20)$ & $0.310(18)$ & $3072.5(15)$ \\
${ }^{26} \mathrm{Al}{ }^{m}$ & $3050.0(11)$ & $1.443(32)$ & $-0.085(15)$ & $0.650(46)$ & $3071.3(21)$ \\
${ }^{34} \mathrm{Cl}$ & $3051.1(10)$ & $1.440(39)$ & $-0.100(15)$ & $0.655(59)$ & $3071.7(24)$ \\
${ }^{38} \mathrm{~K}^{m}$ & $3046.4(14)$ & $1.453(47)$ & $0.035(20)$ & $0.665(56)$ & $3071.2(27)$ \\
${ }^{42} \mathrm{Sc}$ & $3049.6(16)$ & $1.445(54)$ & $-0.035(10)$ & $0.620(63)$ & $3073.4(30)$ \\
${ }^{46} \mathrm{~V}$ & $3044.4(12)$ & $1.445(62)$ & $-0.040(10)$ & $0.655(54)$ & $3066.9(28)$ \\
${ }^{50} \mathrm{Mn}$ & $3047.6(15)$ & $1.443(71)$ & $-0.035(10)$ & $0.770(67)$ & $3066.7(33)$ \\
${ }^{54} \mathrm{Co}$ & $3075.5(14)$ & $1.459(87)$ & $-0.045(20)$ & $1.48(21)$ & $3073.0(72)$ \\
${ }^{62} \mathrm{Ga}$ & $3084.3(80)$ & $1.50(12)$ & $-0.075(30)$ & $1.63(31)$ & $3077(13)$ \\
${ }^{74} \mathrm{Rb}$ & & \multicolumn{5}{c}{ Average $\overline{\mathcal{F} t}$} & $3071.4(8)$ \\
& & \multicolumn{3}{c}{$\chi^{2} / v$} & 0.6 \\
\hline \hline
\end{tabular}

It is clear from the normalized $\chi^{2}$ given on the bottom line of the table that the statistical agreement among the $\mathcal{F} t$ values remains excellent. Furthermore, it is evident from the figure that ${ }^{46} \mathrm{~V}$ no longer shows any deviation from the overall average as it did in Fig. 1. However, it is equally evident that instead the ${ }^{50} \mathrm{Mn}$ and ${ }^{54} \mathrm{Co} \mathcal{F} t$ values are now low, and by amounts that are no less statistically significant than the amount by which the ${ }^{46} \mathrm{~V}$ value was previously high.

Rather than being a negative result, however, this possible discrepancy offers us the opportunity to use the cases of ${ }^{50} \mathrm{Mn}$ and ${ }^{54} \mathrm{Co}$ as a valuable test of our improved calculations. The $Q_{\text {EC }}$ value for each of them has been measured only twice with (claimed) high precision $[48,49]$, and one of these references [48] also included a measurement of the $Q_{\mathrm{EC}}$ value for ${ }^{46} \mathrm{~V}$, which Penning-trap measurements have recently shown $[6,7]$ to be low by $2 \mathrm{keV}$-more than three times its originally quoted standard deviation. If, as seems likely, the problem with the ${ }^{46} \mathrm{~V}$ measurement in Ref. [48] is not limited to that measurement alone, then doubt is certainly cast on the ${ }^{50} \mathrm{Mn}$ and ${ }^{54} \mathrm{Co} Q_{\mathrm{EC}^{-}}$ value results quoted in that reference as well. New Penningtrap measurements of both $Q_{\mathrm{EC}}$ values are currently in progress [50], and the question should be settled shortly. If the $Q_{\mathrm{EC}}$ values in Ref. [48] prove to have been too low again, then the new Penning-trap measurements will serve to increase the $\mathcal{F} t$ values for ${ }^{50} \mathrm{Mn}$ and ${ }^{54} \mathrm{Co}$ and could well bring them into close agreement with the average $\overline{\mathcal{F} t}$ value. If so, this would add strong support to our new calculations.

The average corrected $\overline{\mathcal{F} t}$ value obtained from our new analysis, $3071.4(8) \mathrm{s}$, is more than one standard deviation lower than the comparable result obtained in our 2005 survey, 3072.7(8) s. If the new measurements do prove to increase the $Q_{\mathrm{EC}}$ values for ${ }^{50} \mathrm{Mn}$ and ${ }^{54} \mathrm{Co}$, then this discrepancy will decrease slightly, but there is no avoiding the fact that the inclusion of some core orbitals in the nuclear-structuredependent correction terms has increased the correction in a number of cases, which in turn leads to a reduction in their $\mathcal{F} t$ values. A significant change in the nuclear model has led to a significant change — but not a revolutionary one-in the average $\overline{\mathcal{F} t}$ value.

The new average $\overline{\mathcal{F} t}$ value yields a new value for $V_{\text {ud }}$ via the equation

$$
V_{\mathrm{ud}}^{2}=\frac{K}{2 G_{F}^{2}\left(1+\Delta_{R}^{V}\right) \overline{\mathcal{F} t}},
$$

where $G_{F}$ is the well-known weak-interaction constant for the purely leptonic muon decay [17]. It has been our practice when using the $\overline{\mathcal{F} t}$ value in this context to add $0.85(85) \mathrm{s}$ to its value to account for possible systematic errors in the treatment of the radial wave function in the calculation of $\delta_{C}$. (This point is discussed in detail in Sec. III C of Ref. [1].) Continuing this practice, we obtain the following result for the up-down element of the CKM matrix:

$$
\left|V_{\mathrm{ud}}\right|=0.97418(26) \text {. }
$$

This result can be compared with the value $0.97380(40)$, which was obtained in 2005 [1]. The new value is (just) within the uncertainty of the previous value and carries an uncertainty that is one-third smaller.

The final step is to combine this new value of $\left|V_{\text {ud }}\right|$ with the other top-row elements of the CKM matrix, $\left|V_{\mathrm{us}}\right|$ and $\left|V_{\mathrm{ub}}\right|$, to test the unitarity of the matrix. Taking the values of the latter two elements from the 2006 Particle Data Group review [17] we obtain the stunning result

$$
\left|V_{\mathrm{ud}}\right|^{2}+\left|V_{\mathrm{us}}\right|^{2}+\left|V_{\mathrm{ub}}\right|^{2}=1.0000 \pm 0.0011 .
$$

Unitarity is fully satisfied with a precision of $0.1 \%$. 


\section{CONCLUSIONS}

We have presented new calculations of the nuclearstructure-dependent corrections to superallowed $0^{+} \rightarrow 0^{+}$ nuclear $\beta$ decay. The calculations incorporate core orbitals in the shell model in cases where independent experimental information indicates that they are required. Where possible, they also make use of effective interactions that have been published since our previous calculation of these correction terms [4]. As in that work, we have included 20 transitions in our calculations: 13 that are by now rather well measured and 7 more that are likely to be accessible to precise measurements in the future.

The agreement among the corrected $\mathcal{F} t$ values for the 13 well-measured cases is very good, although there is a possible small discrepancy for the cases of ${ }^{50} \mathrm{Mn}$ and ${ }^{54} \mathrm{Co}$. A new Penning-trap measurement of the $Q_{\text {EC }}$ values for these two transitions is expected in the near future, and its effect on this discrepancy could serve to test the validity of our calculations.

With our new corrections, the value of $\left|V_{\mathrm{ud}}\right|$ is increased by $0.04 \%$, or by one standard deviation of the previous result [1]. With the new value, the sum of squares of the top-row elements of the CKM matrix is in perfect agreement with unitarity. However, this should not be regarded as the end of the story. Although there is excellent agreement with unitarity, it is the $\pm 0.1 \%$ uncertainty on the experimental sum that actually sets the critical limit on possible new physics beyond the standard model. This uncertainty can still be reduced by new precise experiments.

The improved calculations presented in this work were inspired by the remarkable recent improvements in experimental precision, particularly in the measurement of the ${ }^{46} \mathrm{~V} Q_{\mathrm{EC}}$ value. The only way that the calculated corrections can be tested and improved is by such precise measurements, both on the currently well-known transitions and on other as-yetunstudied superallowed transitions that have larger calculated corrections. If the calculated correction terms continue to replace the significant scatter in the measured $f t$ values (see the left panel in Fig. 1) with a set of self-consistent corrected $\mathcal{F} t$ values, then they can surely be relied upon to produce a secure value for $\left|V_{\mathrm{ud}}\right|$ with reduced uncertainties. The present calculations testify to the value of increased experimental precision.

Clearly, precise measurements of superallowed transitions should continue to have high priority. The recent Penning-trap $Q_{\text {EC-value measurements [6,7] that played such an important }}$ role here should be extended to other cases so that ultimately all superallowed transitions will have been measured this way. Furthermore, the superallowed decays of $T_{z}=-1$ nuclei, such as ${ }^{34} \mathrm{Ar}$ and ${ }^{38} \mathrm{Ca}$, have large correction terms and can provide real validation of the accuracy of the calculations if they can be measured with uncertainties comparable to the currently well-known cases; such nuclei will require precise half-lives and branching ratios as well as $Q_{\mathrm{EC}}$ values.

From the point of view of theory, it would also be valuable to have an up-to-date and complete set of nucleus-dependent correction terms based on some different technique from the one used here, perhaps along the lines of the Hartree-Fock calculations employed in the past by Ormand and Brown [5,31, 32]. Even more important, though, is to seek improvements in the precision of the inner radiative correction $\Delta_{R}^{V}$. It continues to be the dominant contributor to the uncertainty quoted for $V_{\mathrm{ud}}$.

\section{ACKNOWLEDGMENTS}

I.S.T. would like to thank the Cyclotron Institute of Texas A\&M University for its hospitality during annual two-month summer visits. The work of J.C.H. was supported by the US Department of Energy under Grant DE-FG03-93ER40773 and by the Robert A. Welch Foundation under Grant A-1397.
[1] J. C. Hardy and I. S. Towner, Phys. Rev. C 71, 055501 (2005).

[2] J. C. Hardy and I. S. Towner, Phys. Rev. Lett. 94, 092502 (2005).

[3] J. C. Hardy, arXiv:hep-ph/0703165v1.

[4] I. S. Towner and J. C. Hardy, Phys. Rev. C 66, 035501 (2002).

[5] W. E. Ormand and B. A. Brown, Phys. Rev. C 52, 2455 (1995).

[6] G. Savard, F. Buchinger, J. A. Clark, J. E. Crawford, S. Gulick, J. C. Hardy, A. A. Hecht, J. K. P. Lee, A. F. Levand, N. D. Scielzo, H. Sharma, K. S. Sharma, I. Tanihata, A. C. C. Villari, and Y. Wang, Phys. Rev. Lett. 95, 102501 (2005).

[7] T. Eronen, V. Elomaa, U. Hager, J. Hakala, A. Jokinen, A. Kankainen, I. Moore, H. Penttilä, S. Rahaman, J. Rissanen, A. Saastamoinen, T. Sonoda, J. Äystö, J. C. Hardy, and V. S. Kolhinen, Phys. Rev. Lett. 97, 232501 (2006).

[8] W. J. Marciano and A. Sirlin, Phys. Rev. Lett. 96, 032002 (2006).

[9] I. S. Towner and J. C. Hardy, Phys. Rev. C 72, 055501 (2005).

[10] B. Hyland, D. Melconian, G. C. Ball, J. R. Leslie, C. E. Svensson, P. Bricault, E. Cunningham, M. Dombsky, G. F. Grinyer, G. Hackman, K. Koopmans, F. Sarazin, M. A. Schumaker, H. C. Scraggs, M. B. Smith, and P. M. Walker, J. Phys. G: Nucl. Part. Phys. 31, S1885 (2005).
[11] T. Eronen, V. Elomaa, U. Hager, J. Hakala, A. Jokinen, A. Kankainen, I. Moore, H. Penttiliä, S. Rahaman, S. Rinta-Antilla, A. Saastamoinen, T. Sonoda, J. Äystö, A. Bey, B. Blank, G. Canchel, C. Dossat, J. Giovinazzo, I. Matea, and N. Adimi, Phys. Lett. B636, 191 (2006).

[12] G. Bollen, D. Davies, M. Facina, J. Huikari, E. Kwan, P. A. Lofy, D. J. Morrissey, A. Prinke, R. Ringle, J. Savory, P. Schury, S. Schwarz, C. Sumithrarachchi, T. Sun, and L. Weissman, Phys. Rev. Lett. 96, 152501 (2006).

[13] P. H. Barker and A. P. Byrne, Phys. Rev. C 73, 064306 (2006).

[14] V. E. Iacob, J. C. Hardy, J. F. Brinkley, C. A. Gagliardi, V. E. Mayes, N. Nica, M. Sanchez-Vega, G. Tabacaru, L. Trache, and R. E. Tribble, Phys. Rev. C 74, 055502 (2006).

[15] B. Hyland, C. E. Svensson, G. C. Ball, J. R. Leslie, T. Achtzehn, D. Albers, C. Andreoiu, P. Bricault, R. Churchman, D. Cross, M. Dombsky, P. Finlay, P. E. Garrett, C. Geppert, G. F. Grinyer, G. Hackman, V. Hanemaayer, J. Lassen, J. P. Lavoie, D. Melconian, A. C. Morton, C. J. Pearson, M. R. Pearson, A. A. Phillips, M. A. Schumaker, M. B. Smith, I. S. Towner, J. J. Valiente-Dobón, K. Wendt, and E. F. Zganjar, Phys. Rev. Lett. 97, 102501 (2006). 
[16] J. T. Burke, P. A. Vetter, S. J. Freedman, B. K. Fujikawa, and W. T. Winter, Phys. Rev. C 74, 025501 (2006).

[17] W.-M. Yao et al., J. Phys. G: Nucl. Part. Phys. 33, 1 (2006).

[18] J. B. French and M. H. Macfarlane, Nucl. Phys. 26, 168 (1961).

[19] D. D. Borlin, Thesis, Washington University, 1967, recorded in the nuclear data sheets of the National Nuclear Data Center: www.nndc.bnl.gov.

[20] I. S. Towner, J. C. Hardy, and M. Harvey, Nucl. Phys. A284, 269 (1977).

[21] S. Cohen and D. Kurath, Nucl. Phys. 73, 1 (1965).

[22] E. K. Warburton and B. A. Brown, Phys. Rev. C 46, 923 (1992).

[23] B. H. Wildenthal, in Progress in Particle and Nuclear Physics, edited by D. H. Wilkinson (Pergamon, Oxford, 1984), Vol. 11, p. 5.

[24] B. A. Brown and W. A. Richter, Phys. Rev. C 74, 034315 (2006).

[25] T. T. S. Kuo and G. E. Brown, Nucl. Phys. 85, 40 (1966).

[26] A. Poves and A. P. Zuker, Phys. Rep. 70, 235 (1981).

[27] W. A. Richter, M. G. van der Merwe, R. E. Julies, and B. A. Brown, Nucl. Phys. A523, 325 (1991).

[28] M. Honma, T. Otsuka, B. A. Brown, and T. Mizusaki, Phys. Rev. C 65, 061301(R) (2002).

[29] M. Honma, T. Otsuka, B. A. Brown, and T. Mizusaki, Phys. Rev. C 69, 034335 (2004).

[30] D. J. Millener and D. Kurath, Nucl. Phys. A255, 315 (1975).

[31] W. E. Ormand and B. A. Brown, Nucl. Phys. A440, 274 (1985).

[32] W. E. Ormand and B. A. Brown, Phys. Rev. Lett. 62, 866 (1989).

[33] J. Britz, A. Pape, and M. S. Antony, At. Data Nucl. Data Tables 69, 125 (1998).

[34] E. Hagberg, V. T. Koslowsky, J. C. Hardy, I. S. Towner,
J. G. Hykawy, G. Savard, and T. Shinozuka, Phys. Rev. Lett. 73, 396 (1994).

[35] W. W. Daehnick and R. D. Rosa, Phys. Rev. C 31, 1499 (1985).

[36] A. Piechaczek, E. F. Zganjar, G. C. Ball, P. Bricault, J. M. D’Auria, J. C. Hardy, D. F. Hodgson, V. Iacob, P. Klages, W. D. Kulp, J. R. Leslie, M. Lipoglavsek, J. A. Macdonald, H.-B. Mak, D. M. Moltz, G. Savard, J. von Schwarzenberg, C. E. Svensson, I. S. Towner, and J. L. Wood, Phys. Rev. C 67, 051305(R) (2003).

[37] A. Sirlin, Phys. Rev. C 164, 1767 (1967).

[38] W. J. Marciano and A. Sirlin, Phys. Rev. D 29, 75 (1984).

[39] W. J. Marciano and A. Sirlin, Phys. Rev. Lett. 56, 22 (1986).

[40] A. Sirlin, Phys. Rev. D 35, 3423 (1987); A. Sirlin and R. Zucchini, Phys. Rev. Lett. 57, 1994 (1986).

[41] W. Jaus and G. Rasche, Phys. Rev. D 35, 3420 (1987).

[42] A. Sirlin, in Precision Tests of the Standard Electroweak Model, edited by P. Langacker (World Scientific, Singapore, 1994).

[43] W. Jaus and G. Rasche, Phys. Rev. D 41, 166 (1990).

[44] I. S. Towner, Nucl. Phys. A540, 478 (1992).

[45] F. C. Barker, B. A. Brown, W. Jaus, and G. Rasche, Nucl. Phys. A540, 501 (1992).

[46] I. S. Towner, Phys. Lett. B333, 13 (1994).

[47] A. Czarnecki, W. J. Marciano, and A. Sirlin, Phys. Rev. D 70, 093006 (2004).

[48] H. Vonach, P. Glaessel, E. Huenges, P. Maier-Komor, H. Roesler, H. J. Scheerer, H. Paul, and D. Semrad, Nucl. Phys. A278, 189 (1977).

[49] V. T. Koslowsky, J. C. Hardy, E. Hagberg, R. E. Azuma, G. C. Ball, E. T. H. Clifford, W. G. Davies, H. Schmeing, U. J. Schrewe, and K. S. Sharma, Nucl. Phys. A472, 419 (1987).

[50] T. Eronen et al. (private communication). 